\title{
Energy policies for eco-friendly households in Luxembourg - a study based on the LuxHEI model
}

\author{
Stéphane Poncin ${ }^{*}$
}

June 24, 2019

\begin{abstract}
In the Grand Duchy of Luxembourg, the residential building sector is a major energy consumer and greenhouse gases emitter that plays a key role in achieving the country's environmental objectives. The purpose of this paper is to assess the effectiveness of the most important policy instruments in decreasing the final energy consumption and $\operatorname{direct} \mathrm{CO}_{2}$ emissions of Luxembourgish households. In order to conduct this study, we developed the LuxHEI model, which is an enhanced and upgraded version of the well-known French simulation model Res-IRF. This variant has also been adjusted to the particular problems of a small country with growing economy and a quickly increasing population. The LuxHEI model goes beyond standard energy-economy models by incorporating global warming as a decision-making factor. The model outcomes reveal that in 2060, and compared to the no-policy baseline scenario, the most aspirational policy mix enables energy savings of $42 \%$ and an emission mitigation of $60 \%$. However, in none of the projections, the residential building sector meets the national energy and climate targets on time. From the results we can draw the following policy implications: for a significant improvement of the sector's energy efficiency and sufficiency, the implementation of a remediation duty for existing buildings and the tightening of the performance standards for new constructions, together with the application of a national carbon tax, are crucial.
\end{abstract}

Key words: climate targets, energy-economic policy modelling, energy efficiency, emission mitigation, residential building sector

JEL Classification: C10, C63, D10, Q4

\footnotetext{
*Stéphane Poncin: CREA, University of Luxembourg. 162A, Avenue de la Faïencerie, L-1511 Luxembourg. E-mail: stephane.poncin@uni.lu

${ }^{+}$The author would like to thank Louis-Gaëtan Giraudet for his valuable help and his encouragements. He further is very grateful to Luisito Bertinelli and Benteng Zou for having suggested this research and for their constant support in its realisation. The author also expresses his gratitude to Daniel Christnach, Tom Eischen, Tom Haas, Markus Lichtmeß, Marc Meyer, Guillaume Osier, François Peltier, Georges Reding, Erik von Scholz, Olivier Thunus, Michael Tomaszewski, Laurent Winkin and Paul Zeimet for their assistance during various stages of his research. Finally, he appreciated the comments from the $2^{\text {nd }}$ International Summer School in Economic modelling of Environment, Energy and Climate, the $5^{\text {th }}$ International Conference on Applied Theory, Macro and Empirical Finance, and the $38^{\text {th }}$ Edition of International Energy Workshop.
} 


\section{Introduction}

The residential building sector is estimated by the European Commission (2010) and the Intergovernmental Panel on Climate Change (Pachauri et al., 2014) to hold a large and cost-effective potential to save energy and reduce emissions. Despite the fact that this potential has been known for a long time, only a fraction of it is currently exploited (Jaffe and Stavins, 1994). However, given that today's efforts to mitigate the devastating consequences of human-made climate change (Lindner et al., 2010) are largely insufficient to meet the vital goals of the Paris Agreement (Olhoff and Christensen, 2018), ${ }^{1}$ the further exhaustion of this key GHG-emitting sector's potential is now more important than ever. In order for this to be realised, the application of energy policy tools that aim to increase energy efficiency and sufficiency is considered to be crucial (Gillingham et al., 2018).

Therefore, the purpose of this paper is to assess the effectiveness of the most important policy instruments in making Luxembourgish households more eco-friendly, that is, achieving savings in the final space heating energy consumption and direct $\mathrm{CO}_{2}$ emissions. More precisely, we analyse: (1) the ranking of the policy instruments in terms of environmental and economic effectiveness when applied individually; (2) the ways in which the instruments generate savings; (3) how the instruments' effectiveness is affected when applied concurrently; (4) whether the national energy and climate objectives are achievable in the country's residential building sector.

There exists a rich literature on the assessment of various energy policy instruments. ${ }^{2}$ Nonetheless, the one that specifically analyses the environmental and economic effectiveness of such instruments, when they are applied (individually or in combination) to promote energy efficiency in the residential building sector, is relatively scarce. Moreover, to the best of our knowledge, the latter analysis has not yet been performed for Luxembourg; the impacts of policy tools can strongly differ from one country to another (Köppel and Ürge-Vorsatz, 2007). To fill this gap, we built on the work of Giraudet et al. $(2011,2012,2015)$ and designed a significantly enhanced Luxembourgish version LuxHEI (Luxembourgish Households' Energy Indicators model) of the French hybrid energy-economy model Res-IRF (Residential module of IMACLIM-R). Furthermore, Luxembourg has committed itself to meet ambitious energy and climate targets, although in the next decades the country is expected to have good economic growth and to face the largest population growth rate (the population is projected to double until 2060) among all EU Member States (Haas and Peltier, 2017). For this reason, the findings of our paper are useful to Luxembourg's political decision-makers.

Basically, the LuxHEI model is designed as a bottom-up model: technologically powerful but microeconomically rather limited (Hourcade et al., 2006). Since the model's microeconomic weaknesses are compensated by incorporating several 'barriers' to energy efficiency, it is considered a hybrid energy-economy model (Hourcade et al., 2006). Actually, engineering bottom-up models tend to follow the assumption of neoclassical economics: consumers behave efficiently when making energy conservation investments. As this hypothesis requires a correct modelling of the costs and the decision-making behaviour, Giraudet et al. (2012) modelled the impacts of 'market barriers', for example, hidden-costs and consumer heterogeneity. However,

\footnotetext{
${ }^{1}$ The central goal of the Paris Agreement of 2015, who was ratified by most parties of the United Nations Framework Convention on Climate Change (UNFCCC), is to keep the rise in global temperatures below 2 degrees Celsius and even to aim for 1.5 degrees Celsius.

${ }^{2}$ Just to mention a few: Weitzman (1974); Pizer (2002); Lee and Yik (2004); Bovenberg et al. (2005); Boonekamp (2006); Geller et al. (2006); Böhringer et al. (2008); Fankhauser et al. (2010); Boeters and Koornneef (2011); Flues et al. (2014); Knobloch et al. (2019); Bye et al. (2018).
} 
in the real world decision-making does not always coincide with the neoclassical standpoint, that is, not all capital expenditures with positive net present value are realised. This phenomenon is often referred to as the energy efficiency gap or paradox. Several attempts to explain this gap appear in the literature (Jaffe and Stavins, 1994; Weber, 1997; Sorrell et al., 2000; Rohdin and Thollander, 2006; Schleich and Gruber, 2008; Thollander and Ottosson, 2008; Fleiter et al., 2011; Trianni and Cagno, 2012). For economists, suboptimal decisions result from an imperfect market structure; in a perfect market consumers would still act rationally (Gillingham and Palmer, 2014). To include this 'market failure', the model takes into consideration asymmetric information, learning-by-using and the principal-agent problem. Unlike neoclassical approaches, behavioural ones assume that 'behavioural failures' lead consumers to make less cost-efficient investments (Gillingham and Palmer, 2014). To account for such suboptimal decision-making, the model relies on restricted consumer awareness. What is more, to reduce overestimations of the sector's energy saving potential, the rebound effect is encoded in the model.

The most important innovative feature of the LuxHEI model is probably the fact that it encodes climate change as a decision-making influence factor. More specifically, we assume that the significant consequences of global warming imply, firstly, that the percentage of the existing building stock that is renovated annually increases over time (from $1 \%$ to $3 \%$ ), and, secondly, that the market becomes more heterogeneous. The model thus goes beyond standard models which are often based on financial considerations only. Indeed, models that do not take sufficient account of the effects of climate change have reduced informative value and misinform policy-makers. The LuxHEI model also changes various modelling methods of the Res-IRF model or adapts them to the available national data, and to the peculiarities of a small country with growing economy and quickly growing population. We encoded for example, the special national situation in all calibration procedures, parameterisations and evaluated policy instruments. ${ }^{3}$ Beyond that, we included more sustainable energy efficiency classes (Zero Energy Buildings (ZEB) and Positive Energy Buildings (PEB)), energy carriers (pellets and solar), and heating systems (heat pumps). As to the carriers considered in the LuxHEI model, some of them can be authorised only in higher energy efficiency classes and some carrier switches are prohibited. We incorporate the discrepancy that exists between the Luxembourgish households' conventional and the effective energy needs for heating through an adjustment factor, which we determined empirically - for each energy efficiency class and each carrier. Further, when an owner retrofitted his dwelling, the tenant or potential buyer profits from reduced heating energy costs. The LuxHEI model encodes the corresponding green value, that is, the percentage of the energy costs savings that the owner can expect recovering through the monthly rent or the sales price. In addition, we encoded a dynamic evolution of the new constructions' building types and changed the inclusion of discount-rates.

As regards the main results of this paper, they can be summarised as follows: (1) the highest environmental and economic effectiveness is achieved by the building codes, followed by the national carbon tax and the remediation duty; (2) while subsidy schemes and regulatory policies have a stronger impact on energy efficiency than on energy sufficiency, it is the other way round for taxes; (3) when the policies are applied concurrently, their individual effects are summed, so that the greatest savings are realised by the policy package with the largest number of instruments; (4) even in the projection with the highest environmental effectiveness, the residential building stock can only meet Luxembourg's energy and climate targets with delay.

\footnotetext{
${ }^{3}$ We wish to emphasise that the LuxHEI model allows to perform the present study for any other country; as long as there exists sufficient data to complete the necessary calibration and parameterisation procedures.
} 
The article is structured as follows. Section 2 describes how the final energy consumption of Luxembourgish households is encoded in the LuxHEI model. In the ensuing section we outline the modelling of the existing building stock's transformation. Section 4 completes the explanation of the model by elucidating the dynamics of the new building stock. The following section depicts the policy instruments that are analysed, as well as the way in which they are modelled. In Section 6, we present and discuss the results of our simulations. In a final section, we draw conclusions and make policy recommendations.

\section{Description of the LuxHEI model (I) - Energy Consumption}

Our objective is to study between 2014 and 2060 the impact of various policy tools on the space heating final energy consumption $E_{\text {fin }}$ of Luxembourgish households. ${ }^{4}$

\subsection{Final energy consumption}

The final energy consumption $E_{\mathrm{fin}}(t)$ in the year $t$ (in $\mathrm{kWh}$ ) is given by

$$
E_{\mathrm{fin}}(t)=S(t) \frac{E_{\mathrm{con}}(t)}{S(t)} \frac{E_{\mathrm{fin}}(t)}{E_{\mathrm{con}}(t)},
$$

where $S(t)$ denotes the total residential building stock (in $\left.m^{2}\right), E_{\text {con }}(t) / S(t)$ is the theoretically/conventionally needed final energy (in $\mathrm{kWh} / \mathrm{m}^{2}$ ), and $E_{\mathrm{fin}}(t) / E_{\mathrm{con}}(t)$ is the quotient of the effective and the conventional needs (dimensionless).

We attribute an energy efficiency class to each dwelling. For existing dwellings we use classes $i \in \mathcal{I}=\{I, \ldots, B, A\}$, where $A$ is the most efficient and $I$ the least efficient of the 9 classes in $\mathcal{I}$. For new buildings we consider only 4 classes $j \in \mathcal{J}=\{B, A, \mathrm{ZEB}, \mathrm{PEB}\}$. We introduce 32 additional categories. Firstly, we distinguish between owner-occupied individual houses and flats and tenant-occupied individual houses and flats, which defines the 4 categories $D \in \mathcal{D}=\{\mathrm{O}-\mathrm{H}, \mathrm{O}-\mathrm{F}, \mathrm{T}-\mathrm{H}, \mathrm{T}-\mathrm{F}\}$. We allow for the 8 energy carriers heating oil, gas, electricity, pellets, oil combined with a solar thermal system, gas with solar, electricity with solar and pellets with solar, which gives the 8 categories $e \in \mathcal{E}=\{\mathrm{F}, \mathrm{G}, \mathrm{E}, \mathrm{P}, \mathrm{F}+\mathrm{s}, \mathrm{G}+\mathrm{s}, \mathrm{E}+\mathrm{s}, \mathrm{P}+\mathrm{s}\}$. Altogether we obtain for each $k \in \mathcal{I} \cup \mathcal{J}$ a $4 \times 8$ matrix of categories $D$, e. Each class $k$ is defined by an overall primary energy demand that we can transform for each type $e$ of carrier into a conventional final energy $\rho_{k, e}$ needed for heating per square meter (and year). ${ }^{5}$ When we denote the residential building stock in $k, D, e$ by $S_{k, D, e}(t)$ and the factor $E_{\mathrm{fin}, k, e}(t) / E_{\mathrm{con}, k, e}(t)$ in $k, e$ by $F_{k, e}(t)$, the final space heating energy of equation (1) is computed by

$$
E_{\mathrm{fin}}(t)=\sum_{\substack{k \in \mathcal{I} \cup \mathcal{J} \\ D \in \mathcal{D} \\ e \in \mathcal{E}}} S_{k, D, e}(t) \rho_{k, e} F_{k, e}(t),
$$

where the dimensionless factor $F_{k, e}(t)$ is the adjustment factor, and where the conventional energies $\rho_{k, e}(k \in\{\mathrm{ZEB}, \mathrm{PEB}\}$ and $e=\mathrm{E}+\mathrm{s})$ are the sums of the buildings' theoretical energy consumption $\rho_{k, e}^{\text {con }}$ and the opposite $\rho_{k, e}^{\text {pro }}<0$ of their theoretical energy production. We highlight

\footnotetext{
${ }^{4}$ Starting from the situation on December 31 of our initial year $t=2013$, we compute the situation on December 31 of the year $t+1=2014$, from this, the situation in 2015, and so on, up to 2060 .

${ }^{5} \mathrm{~A}$ detailed description of the determination of the conventional unit space heating energies can be found in Poncin (2019).
} 
that for $k \in\{\mathrm{ZEB}, \mathrm{PEB}\}, D \in \mathcal{D}$ and for $e=\mathrm{E}+\mathrm{s}$, the terms $S_{k, D, e}(t) \rho_{k, e} F_{k, e}(t)$ in (2) must be interpreted as $S_{k, D, e}(t)\left(\rho_{k, e}^{\text {con }} F_{k, e}(t)+\rho_{k, e}^{\text {pro }}\right)$. In this case we must thus rewrite equation (2) as

$$
E_{\text {fin }}(t)=\sum_{\substack{k \in \mathcal{I} \cup \mathcal{J} \\ D \in \mathcal{D} \\ e \in \mathcal{E}}} S_{k, D, e}(t)\left(\rho_{k, e}^{\text {con }} F_{k, e}(t)+\rho_{k, e}^{\text {pro }}\right) .
$$

\subsection{Adjustment factor}

We model the adjustment factor ${ }^{6}$ as a logistic function

$$
F_{k, e}(t)=a+\frac{b}{1+\exp \left(c \rho_{k, e} P_{e}(t)-d\right)} \quad(a, b, c, d \text { constant }) .
$$

The references for the price $P_{e}(t)$ of the carrier $e$ in the year $t$ (expressed in $€$ per $\mathrm{kWh}$ ) are the energy price projections in Capros et al. (2016) and Birol et al. (2010). The modelling of the adjustment factor by such a logistic function was suggested in Cayre et al. (2011) and used in Giraudet et al. (2012).

Equation (3) captures the impact of energy efficiency measures and energy price variations on energy sufficiency; known as prebound or rebound effect (Sunikka-Blank and Galvin, 2012). Actually, in dwellings with a low energy performance (high $\rho_{k, e}$ ), households tend to consume less energy than the conventional energy $\rho_{k, e}$ (prebound effect, $F_{k, e}(t)<1$ ). The exact opposite can be observed in buildings with a high energy performance (low $\rho_{k, e}$ ): the households' measured energy consumption is close to or even exceeds $\rho_{k, e}$ (rebound effect, $F_{k, e}(t)>1$ ). The user behaviour is similar when $P_{e}(t)$ passes from high values to low ones. The best modelling choice for $F_{k, e}(t)$ is therefore a decreasing logistic function $(c>0)$ in $\rho_{k, e} P_{e}(t)$.

We wish to highlight that the adjustment factor depends on

$$
\rho_{k, e} P_{e}(t)=\left(\rho_{k, e}^{\mathrm{con}}+\rho_{k, e}^{\mathrm{pro}}\right) P_{e}(t)
$$

and not on $\rho_{k, e}^{\text {con }} P_{e}(t)$ alone. In other words we assume that the households adjust their behaviour to the net amount of money that they earn-and not to the money that they spend for heating. Moreover using the latter sum of money would mean that the factor $F_{k, e}(t)$ is the same in the classes $A, \mathrm{ZEB}$ and PEB; since these classes have the same insulation and therefore the same theoretical energy consumption. Our hypothesis implies that the adjustment factor increases when one passes from $A$ to ZEB and from there to PEB.

\section{Description of the LuxHEI model (II) - Dynamics of the existing building stock}

Note that we study separately the building stock that existed at the end of 2013 (EBS) and the building stock that was newly constructed as of 2014 (NBS).

For each $i \in \mathcal{I}, D \in \mathcal{D}$ and $e_{i} \in \mathcal{E}$ (subscript $i$ added to avoid possible subsequent ambiguity), we must compute the existing building stock $S_{i, D, e_{i}}(\tau)$ in $\tau=t+1$ from the known

${ }^{6}$ This factor takes into account the discrepancy that exists between the effective and the conventional energy needs. Additional information about the origin of this discrepancy, as well as a detailed explanation of the adjustment factor's calibration, can be found in Poncin (2019). 
entries $S_{\iota, \Delta, \varepsilon_{\iota}}(t)$ of a $9 \times 4 \times 8$ matrix. We use the formula

$$
S_{i, D, e_{i}}(\tau)=\left(1-\gamma_{i, D, e_{i}}(t)\right) S_{i, D, e_{i}}(t)-\sum_{f>i} \operatorname{TRANS}_{i, f} ; D, e_{i}(\tau)+\sum_{\varphi<i} \operatorname{TRANS}_{\varphi, i} ; D, e_{i}(\tau) .
$$

Here $\gamma_{i, D, e_{i}}(t)$ is the demolition rate of the stock $S_{i, D, e_{i}}(t)$. The second and third terms are the renovations/transitions in $\tau$ from class $i$ to a higher efficiency class $f$, and from a lower class $\varphi$ to the class $i$ respectively. For example, to get the existing stock in class $i=F$ in 2017, we start from the existing stock in class $F$ in 2016 that was not destroyed. From this stock we deduct the buildings in the energy class $F$ that were upgraded to any higher energy class in 2017, and we add the buildings that were upgraded to the energy class $F$ in 2017.

The next formula explains the computation of the second term of equation (4) (the third term is calculated analogously):

$$
\operatorname{TRANS}_{i, f ; D, e_{i}}(\tau)=\left(1-\gamma_{i, D, e_{i}}(t)\right) S_{i, D, e_{i}}(t) \mathrm{X}_{i, D, e_{i}}(\tau) \operatorname{PR}_{i, f ; D, e_{i}}(\tau)
$$

This means that in order to find the transitions/retrofits from the initial class $i$ to any higher final class $f$, the model first computes the fraction $\mathrm{X}_{i, D, e_{i}}(\tau)$ of the nondestroyed stock in $i$ that is retrofitted in $\tau$ (proportion of retrofits in class $i$ ), then the fraction $\mathrm{PR}_{i, f ; D, e_{i}}(\tau)$ of the latter that is retrofitted to $f$ in $\tau$ (proportion of retrofits to class $f$ ). We explain in Section 3.5 how the demolition rate $\gamma_{i, D, e_{i}}(t)$ is computed from the time-invariant average demolition rate $\gamma$ in the whole stock $S(t)$.

\subsection{Distributions of retrofits}

The distribution $\mathrm{PR}_{i, f} ; D, e_{i}(\tau)$ in the year $\tau$ of decided retrofits in a class $i$ over all higher classes $f$ is calculated by

$$
\operatorname{PR}_{i, f ; D, e_{i}}(\tau)=\frac{\operatorname{LCC}_{i, f, D, e_{i}}(\tau)^{-\nu}}{\sum_{h>i} \operatorname{LCC}_{i, h, D, e_{i}}(\tau)^{-\nu}}
$$

Indeed, when a retrofit from $i$ was decided, the number of retrofits from $i$ to $f$ is roughly proportional to the inverse of the life cycle costs $\mathrm{LCC}_{i, f, D, e_{i}}(\tau)$ of such a renovation. Hence, the percentage $\mathrm{PR}_{i, f ; D, e_{i}}(\tau)$ is obtained by equation (6) with $\nu=1$. However, the percentages observed in the initial year do not correspond well with the computed ones. The accordance becomes better for higher values of $\nu$ and the best one is obtained for $\nu=7$. This technique was introduced by Jaccard and Dennis (2006) to model consumer heterogeneity, which corresponds to a market barrier. Values of $\nu$ close to 1 reflect preference heterogeneity: the choice of different investment options is relatively even. In contrast higher values of $\nu$ such as $\nu=7$ reflect a more homogeneous investment behaviour: the retrofitting option with the lowest life cycle costs $\mathrm{LCC}_{i, h, D, e_{i}}(\tau)$ is selected by most consumers.

\section{Climate change}

Up to here the model is based on the price-demand relationship and ignores possible shocks that could suspend this rule. In consideration of current climate trends (Lindner et al., 2010), it is likely that over the next decades, the effects of climate change become more and more perceptible for society. In addition, within the modelling period, not only will the Luxembourgish population's educational level keep raising (Schofer and Meyer, 2005), but the country is 
also projected to face economic growth and increasing disposable household incomes (Haas and Peltier, 2017). Notice that environmental awareness is an increasing function of the experience of global warming impacts (Reynolds et al., 2010) and the educational level (Palmer et al., 1999; Aminrad et al., 2011). Further, based on the findings of Huang et al. (2006), the inhabitants of a territory with economic growth, an above-average income per capita, and bad environmental quality, have great willingness to invest in environmental improvement measures. This tendency is strengthened by self-serving reasons (Huang et al., 2006), for instance, an improvement of the insulation of a dwelling to decrease suffering from heat rather than to protect the climate. With regard to the latter observations, we assume that Luxembourgish households will progressively accept to spend more money for a retrofitting to a low energy class and a nonfossil carrier-even when this decision is financially not really interesting. In other words, the market will become more heterogeneous: the parameter $\nu$ will decrease over time. A detailed description of the modelling method of the dynamic parameter $\nu$ can be found in Appendix A.1.

\section{Life cycle costs}

The life cycle costs $\mathrm{LCC}_{i, f, D, e_{i}}(\tau)$ in (6) are the sum of the investment/retrofitting costs $\operatorname{INVC}_{i, f}(\tau)$, the energy operating costs $\operatorname{ENERC}_{i, f, D, e_{i}}(\tau)$, and the intangible costs $\operatorname{IC}_{i, f}(\tau)$ :

$$
\mathrm{LCC}_{i, f, D, e_{i}}(\tau)=\operatorname{INVC}_{i, f}(\tau)+\operatorname{ENERC}_{i, f, D, e_{i}}(\tau)+\mathrm{IC}_{i, f}(\tau) .
$$

The model assumes that first the decision to renovate from $i$ to $f$ is made and that only then the decision to switch from the initial carrier $e_{i}$ to a final one is taken. Therefore the energy operating costs $\operatorname{ENERC}_{i, f, D, e_{i}}(\tau)$ are based on the initial carrier $e_{i}$. We will explain below their dependence on $i \in \mathcal{I}$ and on $D \in \mathcal{D}$.

\section{(1) Investment costs}

The evolution of the investment costs $\operatorname{INVC}_{i, f}(\tau)$ is modelled by

$$
\operatorname{INVC}_{i, f}(\tau)=\operatorname{INVC}_{i, f}(0)\left(\alpha+(1-\alpha)(1-l)^{\log _{2} \frac{C_{f}(\tau)}{C_{f}(0)}}\right) .
$$

A large spectrum of measures can be taken to retrofit a building from an energy class $i$ to a higher class $f$. The initial retrofitting costs $\operatorname{INVC}_{i, f}(0)$ of Table 1 are therefore average costs, which include costs ranging from small improvements of the envelope and the heating system to significant ones. ${ }^{7}$

\footnotetext{
${ }^{7}$ The matrix of initial investment costs respects similar rules to those used in Giraudet et al. (2012) and was determined after concertation with experts from renovation companies.
} 
Table 1 - Initial retrofitting costs $\operatorname{INVC}_{i, f}(0)$ (in $€ / m^{2}$ )

\begin{tabular}{|c|c|c|c|c|c|c|c|c|}
\cline { 2 - 9 } \multicolumn{1}{c|}{} & $\mathrm{H}$ & $\mathrm{G}$ & $\mathrm{F}$ & $\mathrm{E}$ & $\mathrm{D}$ & $\mathrm{C}$ & $\mathrm{B}$ & $\mathrm{A}$ \\
\hline $\mathrm{I}$ & 100 & 250 & 475 & 775 & 1150 & 1600 & 2125 & 2725 \\
\hline $\mathrm{H}$ & & 190 & 415 & 715 & 1090 & 1540 & 2065 & 2665 \\
\hline $\mathrm{G}$ & & & 280 & 580 & 955 & 1405 & 1930 & 2530 \\
\hline $\mathrm{F}$ & & & & 370 & 745 & 1195 & 1720 & 2320 \\
\hline $\mathrm{E}$ & & & & & 460 & 910 & 1435 & 2035 \\
\hline $\mathrm{D}$ & & & & & & 550 & 1075 & 1675 \\
\hline $\mathrm{C}$ & & & & & & & 640 & 1240 \\
\hline $\mathrm{B}$ & & & & & & & & 730 \\
\hline
\end{tabular}

The idea of equation (8) is that the retrofitting costs $\operatorname{INVC}_{i, f}(0)$ in the year 0 have decreased in the year $\tau$ due to the experience $C_{f}(\tau)$ accumulated in $\tau$ through realised retrofits to the class $f$. The term $\operatorname{INVC}_{i, f}(0) \alpha$ is the percentage $\alpha$ of the initial retrofitting costs that cannot be decreased by experience (see Table 4 for the precise value of $\alpha$ ). The reduction of the remaining costs $\operatorname{INVC}_{i, f}(0)(1-\alpha)$ is modelled through the multiplication by the exponential function $(1-l)^{\log _{2} \frac{C_{f}(\tau)}{C_{f}(0)}}$. The constant $l$ (see Table 4 ) is a market failure called the learning-by-doing rate. The accumulated experience $C_{f}(\tau)$ is calculated from $C_{f}(t)$ by the formulas

$$
C_{f}(\tau)=C_{f}(t)+\sum_{i<f} \sum_{D, e_{i}} \operatorname{TRANS}_{i, f ; D, e_{i}}(t)
$$

and

$$
C_{f}(0)=15 \times 1 \% \times S_{f}(0) .
$$

Note that the experience $C_{f}(0)$ in 2013 was accumulated through retrofits between 1998 and 2012. For $C_{f}(\tau)=2^{n} C_{f}(0)$ we find that

$$
(1-l)^{\log _{2} \frac{C_{f}(\tau)}{C_{f}(0)}}=(1-l)^{n},
$$

for each doubling of the experience the price $\operatorname{INVC}_{i, f}(0)(1-\alpha)$ is hence multiplied by $1-l$, that is, it decreases by $l$.

\section{(2) Energy operating costs}

\section{Step 1: Approximate energy costs}

We used already the energy price projections $P_{e}(t)$ for the carriers $e$ (in $€$ per $\mathrm{kWh}$ ) (Capros et al., 2016; Birol et al., 2010). The energy operating costs (in $€$ per $m^{2}$ ) over the average lifetime $N$ (see Table 4 ) of a retrofit are the sums

$$
\operatorname{ENERC}_{f, e_{i}}(\tau)=\sum_{\mathfrak{t}=1}^{N} P_{e_{i}}(\tau+\mathfrak{t}) \rho_{f, e_{i}} .
$$

The terms of these sums are costs, which we denote $C_{\mathrm{t}}$, that are paid over the $N$ years of the lifetime. The decision maker on the retrofit may use money from an interest-bearing investment with interest rate $r_{D}$. On that account, she bases her decision on the net 
present value of the periodic cash flows $C_{\mathfrak{t}}$. The model also considers the prices $P_{e_{i}}(\tau+\mathfrak{t})$ as constant over lifetime and replaces them by $P_{e_{i}}(\tau)$. The reason behind this modelling choice is a market failure. We assume that similarly to the findings in Simon (1955), the uncertainty about the energy price evolution leads people to drop a part of the information at disposal when making decisions about energy conservation investments. The energy operating costs are thus calculated by

$$
\operatorname{ENERC}_{f, D, e_{i}}(\tau)=P_{e_{i}}(\tau) \rho_{f, e_{i}} \sum_{\mathfrak{t}=1}^{N}\left(1+r_{D}\right)^{-\mathfrak{t}} .
$$

This equation allows one to account for the Landlord-Tenant dilemma, an important market failure to energy renovation in the residential sector of the European Union (Ástmarsson et al., 2013). This principal/agent issue occurs if tenants and landlords have split incentives: tenants wish to reduce their energy bill through energy efficiency measures but owners are reticent to come up for the costs (as they have no direct return on the investment) (Gillingham et al., 2012; Charlier, 2015). As a result, non-occupying homeowners require for investments into energy efficiency higher profitability than occupying homeowners. In order to model the lower (higher) number of renovations in the categories T-H and T-F (O-H and O-F), we assign different interest rates to these four decision situations $D$ :

$$
r_{\mathrm{T}-\mathrm{H}}=0.10, r_{\mathrm{T}-\mathrm{F}}=0.07, r_{\mathrm{O}-\mathrm{H}}=0.30 \text { and } r_{\mathrm{O}-\mathrm{F}}=0.25 \text {. }
$$

The lower (higher) rates for tenant-occupied (owner-occupied) dwellings produce higher (lower) net present values or energy operating costs. The model yields therefore lower (higher) numbers of renovations in the categories T-H and T-F (O-H and O-F). Further, the weighted mean of the four percentages is 0.24 , which agrees with the observation of Hausman (1979) and Train (1985) that the range of this mean is $0.20-0.25$.

\section{Step 2: Energy costs with green value}

In Luxembourg, owners sell their dwellings after an average period $T$ of 9 years. When an owner retrofits (we assume that he renovates right after he bought the habitation), the potential tenant and the future buyer have the advantage of reduced energy costs. The green value $\mathcal{G}$ (see Table 4) is the percentage of the energy cost savings that the owner recovers through monthly rents or an increased sales price. For occupying owners $D$ we replace the approximate energy costs (11) by the energy costs

$$
\begin{aligned}
& \operatorname{ENERC}_{i, f, D, e_{i}}(\tau)= \\
& \qquad P_{e_{i}}(\tau) \rho_{f, e_{i}} \sum_{\mathfrak{t}=1}^{T}\left(1+r_{D}\right)^{-\mathfrak{t}}-\left(P_{e_{i}}(\tau)\left(\rho_{i, e_{i}}-\rho_{f, e_{i}}\right) \sum_{\mathfrak{t}=T+1}^{N}\left(1+r_{D}\right)^{-\mathfrak{t}}\right) \mathcal{G},
\end{aligned}
$$

where the last term is the percentage of the energy cost savings of the new owner which $D$ recovers when selling her dwelling. If $D$ is a nonoccupying owner she can furthermore 
recover the same percentage through the rents that the tenant pays during the first $T$ years:

$$
\begin{aligned}
& \operatorname{ENERC}_{i, f, D, e_{i}}(\tau)= \\
& P_{e_{i}}(\tau)\left(\rho_{f, e_{i}}-\left(\rho_{i, e_{i}}-\rho_{f, e_{i}}\right) \mathcal{G}\right) \sum_{\mathfrak{t}=1}^{T}\left(1+r_{D}\right)^{-\mathfrak{t}}-\left(P_{e_{i}}(\tau)\left(\rho_{i, e_{i}}-\rho_{f, e_{i}}\right) \sum_{\mathfrak{t}=T+1}^{N}\left(1+r_{D^{\prime}}\right)^{-\mathfrak{t}}\right) \mathcal{G},
\end{aligned}
$$

where $r_{D^{\prime}}$ is the owner interest rate that corresponds to the tenant interest rate $r_{D}$ (for example, if $D=\mathrm{T}-\mathrm{H}$, then $D^{\prime}=\mathrm{O}-\mathrm{H}$; since the buyer tries to reduce the increase of the sales price).

\section{(3) Intangible costs}

When the calculation of the proportions $\mathrm{PR}_{i, f} ; D, e_{i}(\tau)$ is based only on the costs $\operatorname{INVC}_{i, f}(\tau)$ and $\operatorname{ENERC}_{i, f, D, e_{i}}(\tau)$, the computed proportions do not coincide in the year 0 with the observed proportions. In order to counter this gap, Giraudet et al. (2012) used intangible costs $\mathrm{IC}_{i, f}(\tau){ }^{8}$ These intangible costs are split into hidden intangible costs $\mathrm{HIC}_{i, f}(\tau)$ (market barrier) and intangible costs $\mathrm{IIC}_{i, f}(\tau)$ due to imperfect information. Hidden costs can hardly be changed and they are therefore calculated as a constant percentage $\beta$ (see Table 4) of the initial intangible costs: $\mathrm{HIC}_{i, f}(\tau)=\mathrm{IC}_{i, f}(0) \beta$. With growing accumulated experience $C_{f}(\tau)$, imperfect information gets smaller (Jaffe and Stavins, 1994; Jaffe et al., 2004), so that the costs $\operatorname{IIC}_{i, f}(\tau)$ decrease and tend finally to disappear completely. We model the evolution of these costs by

$$
\mathrm{IIC}_{i, f}(\tau)=\mathrm{IC}_{i, f}(0) \frac{1}{1+c \exp \left(d \frac{C_{f}(\tau)}{C_{f}(0)}\right)} \quad(c, d>0),
$$

where the decreasing logistic function of the relative accumulated experience $C_{f}(\tau) / C_{f}(0)$ takes the value $1-\beta$ for $\tau=0$. Equation

$$
\mathrm{IC}_{i, f}(\tau)=\mathrm{HIC}_{i, f}(\tau)+\mathrm{IIC}_{i, f}(\tau)=\mathrm{IC}_{i, f}(0)\left(\beta+\frac{1}{1+c \exp \left(d \frac{C_{f}(\tau)}{C_{f}(0)}\right)}\right)
$$

is thus consistent in the year 0 .

Additionally, when the initial accumulated experience ${ }^{9}$ doubles, the initial value $1-\beta$ is multiplied by a factor $1-\mu$. The percentage $\mu$ can be compared with the learning-by-doing rate $l$. Indeed when the initial accumulated experience doubles, the initial value $1-\alpha$ (see above) is multiplied by $1-l$. In other words, the percentage $\mu$ (see Table 4 ) corresponds to the information acceleration rate related to the asymmetric information which causes the market failure. The learning-by-doing rate has an analogous interpretation. We obtain that way the system of equations

$$
\frac{1}{1+c \exp (d)}=1-\beta \text { and } \frac{1}{1+c \exp (2 d)}=(1-\beta)(1-\mu)
$$

\footnotetext{
${ }^{8}$ The idea of using intangible costs to ameliorate the modelling of life cycle costs stems from the energy-economy model CIMS (Jaccard and Dennis, 2006; Rivers and Jaccard, 2005).

${ }^{9}$ The accumulated experience is calculated as before by equation (9) and (10).
} 
where

$$
c=\frac{(1-\mu) \beta^{2}}{(\mu+(1-\mu) \beta)(1-\beta)}>0 \quad \text { and } \quad d=\ln \left(\frac{\mu}{(1-\mu) \beta}+1\right)>0 .
$$

The constant $c$ determines the proportion $1 /(1+c)$ that corresponds to $C_{f}(\tau)=0$ and the constant $d$ is responsible for the steepness of the sigmoid curve. Equation (15) shows that if the information acceleration rate $\mu$ increases, the values of $1 /(1+c)$ and $d$ increase; just the way it should be.

We can use equation (14) once the initial intangible costs are known. Because they are intangible, the initial costs $\mathrm{IC}_{i, f}(0)$ cannot be observed but must be calculated. A detailed presentation of the calibration procedure can be found in Appendix A.2.

\subsection{Further description of the transformations of the existing building stock}

We now rewrite equation (5) by incorporating the carrier switch that we mentioned below equation (7), that is, we must compute the transitions from $i$ to $f$ and $e_{i}$ to $e_{f}$. Therefore we calculate the total proportions $\mathrm{PRT}_{i, f ; e_{i}, e_{f} ; D}$. Such a proportion is the product of the proportion $\mathrm{PR}_{i, f ; D, e_{i}}$ of retrofits from $i$ to $f$ and of the conditional proportion $\left.\mathrm{PRS}_{e_{i}, e_{f} ; D}\right|_{i, f}$ of switches from $e_{i}$ to $e_{f}$.

We thus compute the transitions

$\operatorname{TRANS}_{i, f ; e_{i}, e_{f} ; D}(\tau)=$

$$
\left.\left(1-\gamma_{i, D, e_{i}}(t)\right) S_{i, D, e_{i}}(t) \mathrm{X}_{i, D, e_{i}}(\tau) \operatorname{PR}_{i, f ; D, e_{i}}(\tau) \operatorname{PRS}_{e_{i}, e_{f} ; D}\right|_{i, f}(\tau),
$$

$(f>i)$ and the transitions

$$
\begin{aligned}
\operatorname{TRANS}_{\varphi, i} ; e_{\varphi}, e_{i} ; D & (\tau)= \\
& \left.\left(1-\gamma_{\varphi, D, e_{\varphi}}(t)\right) S_{\varphi, D, e_{\varphi}}(t) \mathrm{X}_{\varphi, D, e_{\varphi}}(\tau) \operatorname{PR}_{\varphi, i ; D, e_{\varphi}}(\tau) \operatorname{PRS}_{e_{\varphi}, e_{i} ; D}\right|_{\varphi, i}(\tau),
\end{aligned}
$$

$(\varphi<i)$. In order to obtain the number of transitions (or the corresponding number of square meters) that is needed in (4), we sum the transitions (16) over all $f>i$ and all $e_{f}$, and we sum the transitions (17) over all $\varphi<i$ and all $e_{\varphi}$. The first sum is equal to

$$
\begin{aligned}
& \sum_{f>i} \operatorname{TRANS}_{i, f ; D, e_{i}}(\tau)= \\
& \qquad \begin{array}{r}
\left(1-\gamma_{i, D, e_{i}}(t)\right) S_{i, D, e_{i}}(t) \mathrm{X}_{i, D, e_{i}}(\tau) \sum_{f>i} \mathrm{PR}_{i, f} ; D, e_{i} \\
\left.(\tau) \sum_{e_{f}} \operatorname{PRS}_{e_{i}, e_{f} ; D}\right|_{i, f}(\tau)= \\
\left(1-\gamma_{i, D, e_{i}}(t)\right) S_{i, D, e_{i}}(t) \mathrm{X}_{i, D, e_{i}}(\tau) .
\end{array}
\end{aligned}
$$

The second sum is equal to

$$
\begin{aligned}
& \sum_{\varphi<i} \operatorname{TRANS}_{\varphi, i ; D, e_{i}}(\tau)= \\
& \left.\quad \sum_{\varphi<i} \sum_{e_{\varphi}}\left(1-\gamma_{\varphi, D, e_{\varphi}}(t)\right) S_{\varphi, D, e_{\varphi}}(t) \mathrm{X}_{\varphi, D, e_{\varphi}}(\tau) \operatorname{PR}_{\varphi, i ; D, e_{\varphi}}(\tau) \operatorname{PRS}_{e_{\varphi}, e_{i} ; D}\right|_{\varphi, i}(\tau),
\end{aligned}
$$

and really depends on the proportions PR and PRS. 


\subsection{Distributions of carrier switches}

\section{Homogeneous market}

We calculate the (conditional) proportions $\left.\mathrm{PRS}_{e_{i}, e_{f} ; D}\right|_{i, f}(\tau)$ of switches from $e_{i}$ to $e_{f}$ analogously to the proportions $\mathrm{PR}_{i, f} ; D, e_{i}(\tau)$ of retrofits from $i$ to $f$ :

$$
\left.\operatorname{PRS}_{e_{i}, e_{f} ; D}\right|_{i, f}(\tau)=\frac{\operatorname{LCCS}_{f, D, e_{i}, e_{f}}^{-\nu(\tau)}(\tau)}{\sum_{e_{h}} \operatorname{LCCS}_{f, D, e_{i}, e_{h}}^{-\nu(\tau)}(\tau)}
$$

Here $\nu(\tau)$ is the dynamic heterogeneity parameter (given by equation (29) of Appendix A.1) and

$$
\operatorname{LCCS}_{f, D, e_{i}, e_{f}}(\tau)=\operatorname{SWIC}_{e_{i}, e_{f}}+P_{e_{f}}(\tau) \rho_{f, e_{f}} \sum_{\mathfrak{t}=1}^{M}\left(1+r_{D}\right)^{-\mathfrak{t}} .
$$

The life cycle costs (21) of a switch from $e_{i}$ to $e_{f}$ are similar to the life cycle costs (7) of a retrofit from $i$ to $f$. The switching costs ${ }^{10} \mathrm{SWIC}_{e_{i}, e_{f}}$ of equation (21) include costs arising from oil tank removal, drilling for geothermal probes or laying a gas pipe, corresponding services provided by electricians or masons, etc., while the analogous investment $\operatorname{costs} \mathrm{INVC}_{i, f}(\tau)$ in equation (7) include the heater and heater installation costs. The second term of (21) can be compared with the term $\operatorname{ENERC}_{i, f, D, e_{i}}$ of (7), except that in the present situation the final carrier is known and that we can compute therefore the energy costs using this carrier (which is more natural). Whereas the lifetime of a retrofit is $N$ years, the lifetime of a carrier switch is $M$ years (see Table 4). We do not use a green value in (21) because we switch the carrier in a fixed efficiency class. In contrast with (7), equation (21) does not contain intangible costs since in Luxembourg the observations needed for the calibration of the initial intangible costs are unavailable. Finally the switching costs are considered as constant, no learning effect is included, also due to infeasibility.

As illustrated in Table 2, the final carriers 'pellets' $(\mathrm{P})$, 'pellets combined with a solar thermal system' $(\mathrm{P}+\mathrm{s})$, 'electricity' $(\mathrm{E})$, and 'electricity with solar' $(\mathrm{E}+\mathrm{s})$ can be chosen only in higher energy efficiency classes. Firstly, we mentioned earlier that each energy efficiency class is initially defined in primary energy $Q_{\text {pri, }}$ and then transformed in the model for each type of carrier into final energy $Q_{\text {fin }}$. Based on the data of the Ministry of the Economy of Luxembourg (2017), we find that the final energy of almost all Luxembourgish dwellings is lower than $643 \mathrm{kWh} / \mathrm{m}^{2} /$ year. Consequently, when a person who renovates chooses the final carrier $\mathrm{P}$ or $\mathrm{P}+\mathrm{s}$, the primary energy $Q_{\mathrm{pri}}=0.07 Q_{\mathrm{fin}}$ is lower than 45 . This, however, does mean that the dwelling has the energy efficiency class $A$. In other words, a person who renovates to the final class $f=C$ or $f=B$ cannot choose the carriers $\mathrm{P}$ and $\mathrm{P}+\mathrm{s}$, otherwise it misses almost always its goal to renovate to $f$. On this account our model allows the choice of the carriers $\mathrm{P}$ and $\mathrm{P}+\mathrm{s}$ only if the chosen final class is $f=A$ (see Table 2). Secondly, given the bad overall efficiency of electric heaters and the resulting environmental disadvantages, the Luxembourgish government wants to push back these heating systems and promotes the use of heat pumps instead. Hence in our model, if $e_{f}=\mathrm{E}$ or $e_{f}=\mathrm{E}+\mathrm{s}$, the heating system used is a heat pump. However, for technical reasons, heat pumps are only adapted for space heating in the energy classes $B, A$, ZEB and PEB (Myenergy Luxemgourg, 2018). This is why carrier switches to $e_{f} \in\{\mathrm{E}, \mathrm{E}+\mathrm{s}\}$ are only permitted if $f>C$ (see Table 2 ).

\footnotetext{
${ }^{10}$ These costs were determined after concertation with experts from renovation companies.
} 
Table 2 - Switching costs $\mathrm{SWIC}_{e_{i}, e_{f}}$ (in $€ / m^{2}$ )

\begin{tabular}{|c|c|c|c|c|c|c|c|c|c|c|c|c|}
\hline & Oil & Gas & $\begin{array}{c}\text { Electricity } \\
(\text { if } \mathrm{k}<\mathrm{B} \text { ) }\end{array}$ & $\begin{array}{l}\text { Electricity } \\
\text { (if } \mathrm{k}>=\mathrm{B})\end{array}$ & $\begin{array}{c}\text { Pellets } \\
\text { (if } \mathrm{k}<\mathrm{A} \text { ) }\end{array}$ & $\begin{array}{c}\text { Pellets } \\
(\text { if } \mathrm{k}=\mathrm{A})\end{array}$ & $\begin{array}{l}\text { Oil }+ \\
\text { Solar }\end{array}$ & $\begin{array}{c}\text { Gas }+ \\
\text { Solar }\end{array}$ & $\begin{array}{c}\text { Electricity } \\
+ \text { Solar } \\
(\text { if } \mathrm{k}<\mathrm{B})\end{array}$ & $\begin{array}{c}\text { Electricity } \\
+ \text { Solar } \\
\text { (if } \mathrm{k}>=\mathrm{B} \text { ) }\end{array}$ & $\begin{array}{c}\begin{array}{c}\text { Pellets } \\
\text { + Solar } \\
(\text { if } \mathrm{k}<\mathrm{A})\end{array} \\
\end{array}$ & $\begin{array}{l}\text { Pellets } \\
+ \text { Solar } \\
\text { (if k=A) }\end{array}$ \\
\hline Oil & 0 & 64 & & 50 & & 82 & 27 & 91 & & 77 & & 109 \\
\hline Gas & 61 & 0 & & 50 & & 82 & 88 & 27 & & 77 & & 109 \\
\hline Electricity & 183 & 186 & 0 & 172 & & 204 & 210 & 213 & & 199 & & 231 \\
\hline Pellets & 61 & 64 & & 50 & 0 & 0 & 88 & 91 & & 77 & 27 & 27 \\
\hline Oil+Solar & & & & & & & 0 & 64 & & 50 & & 82 \\
\hline Gas+Solar & & & & & & & 61 & 0 & & 50 & & 82 \\
\hline Electricity + Solar & & & & & & & 61 & 64 & 0 & 0 & & 82 \\
\hline Pellets+Solar & & & & & & & 61 & 64 & & 50 & 0 & 0 \\
\hline
\end{tabular}

In contrast with what we just said, the heating system of an initial carrier $e_{i} \in\{\mathrm{E}, \mathrm{E}+\mathrm{s}\}$ is an electric heater. As these systems consist mostly of direct-heating electric radiators and not of central heating systems (as do all other carriers in the model), switching from such an $e_{i}$ to any other carrier is very expensive. Finally, because carrier switches are related to retrofits to higher energy classes, households who used already 'solar' do usually not switch to a carrier without 'solar'. This means that switches from $e_{i} \in\{\mathrm{F}+\mathrm{s}, \mathrm{G}+\mathrm{s}, \mathrm{E}+\mathrm{s}, \mathrm{P}+\mathrm{s}\}$ to $e_{f} \in\{\mathrm{F}, \mathrm{G}, \mathrm{E}, \mathrm{P}\}$ are not allowed (see Table 2).

\section{Heterogeneous marked}

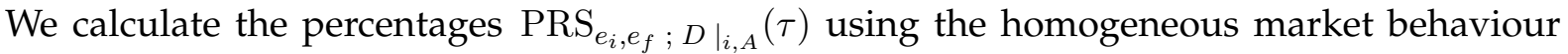
defined by the heterogeneity parameter $\nu(\tau)$. The numbers of houses using $\mathrm{E}, \mathrm{E}+\mathrm{s}, \mathrm{P}$ or $\mathrm{P}+\mathrm{s}$ are then rather low in 2060. The reason of this insufficiency of the model are the values of $\nu(\tau)$ which range from 7 to 3.25 (when the life cycle costs double the percentage of switching decisions deceases from $100 \%$ to a percentage between approximately $1 \%$ and $10 \%)$. The values of $\nu(\tau)$ can of course decrease in specific subpopulations. For example, switches in the class $A$ to one of the carriers $\mathrm{E}, \mathrm{E}+\mathrm{s}, \mathrm{P}$ or $\mathrm{P}+\mathrm{s}$ reflect a very good environmental consciousness, which decreases the effect of costs on the switching decision. In order to remedy the mentioned insufficiency of the model, we decrease $\nu(\tau)$ in the calculation of the proportions $\operatorname{PRS}_{e_{i}, e_{f} ;\left.D\right|_{i, f}}(\tau)$. A complete illustration of this decrease can be found in Appendix A.3.

\subsection{Fraction of retrofitted buildings}

\section{Without climate change}

The proportion $X_{i, D, e_{i}}(\tau)$ of retrofits of dwellings of class $i$ is correlated to the profitability of the corresponding investment. The net present value $\mathrm{NPV}_{i, D, e_{i}}(\tau)$ of such a retrofit is the difference between the lifetime energy costs in the class $i$ (when no retrofit is made) and the weighted average lifetime costs of a retrofit from $i$ to any higher class $f$ :

$$
\operatorname{NPV}_{i, D, e_{i}}(\tau)=\operatorname{ENERC}_{i, D, e_{i}}(\tau)-\sum_{f>i} \operatorname{PR}_{i, f} ; D, e_{i}(\tau) \operatorname{LCC}_{i, f, D, e_{i}}(\tau)
$$


The precise relation between $\operatorname{NPV}_{i, D, e_{i}}(\tau)$ and $X_{i, D, e_{i}}(\tau)$ is modelled by a logistic function: ${ }^{11}$

$$
X_{i, D, e_{i}}(\tau)=\frac{1}{1+a \exp \left(-b \mathrm{NPV}_{i, D, e_{i}}(\tau)\right)} \quad(a, b>0) .
$$

If the net present value begins to increase, it is not yet really attractive and the proportion of retrofits increases only slowly; if the profit of a retrofit becomes more and more attractive the proportion increases quicker.

Equation

$$
\sum_{i, D, e_{i}} \frac{S_{i, D, e_{i}}(0)}{1+a \exp \left(-b \operatorname{NPV}_{i, D, e_{i}}(1)\right)}=0.01 S(0)
$$

asks that the surface which is retrofitted in the first year be $1 \%$ of the surface of the existing building stock in the initial year. The constants $a$ and $b$ are the positive solutions of this equation for which the percentage $1 /(1+a)$ of retrofits for zero-profitability is minimal. This calibration problem is an optimisation problem under constraint and because of that, it is solved numerically using Lagrange multipliers.

\section{With climate change}

The percentage of the existing stock $S(t)$ which is renovated in the next year will increase over time. ${ }^{12}$ This percentage $p(\tau)$ is modelled as an increasing logistic function, ${ }^{13}$ and by applying the same procedure as the one for $\nu(\tau)$ (see Appendix A.3), we obtain

$$
p(\tau)=0.01\left(0.85+\frac{2.30}{1+\exp (-0.124 \tau+2.66)}\right) .
$$

In this modelling alternative $a$ and $b$ in (22) depend on $\tau: a=a_{\tau}$ and $b=b_{\tau}$. Their calculation uses the time-dependent constraint

$$
\sum_{i, D, e_{i}} \frac{S_{i, D, e_{i}}(t)}{1+a_{\tau} \exp \left(-b_{\tau} \operatorname{NPV}_{i, D, e_{i}}(\tau)\right)}=(p(\tau)-0.01) S(t)+\sum_{i, D, e_{i}} \frac{S_{i, D, e_{i}}(t)}{1+a \exp \left(-b \operatorname{NPV}_{i, D, e_{i}}(\tau)\right)}
$$

In this formula the sum at the left hand side is the total surface which is renovated in the year $\tau$ after consideration of the economic and the climatic issues encoded in the LuxHEI model. The percentage $p(\tau)$ in the right hand side increases from the current $1 \%$ to $3 \%$ due to (essentially) climatic reasons. Since in the term $(p(\tau)-0.01) S(t)$ we subtract the approximate total surface $0.01 S(t)$ that is renovated in $\tau$ for economic reasons, this term represents the total surface renovated in $\tau$ for climatic reasons. Adding the last term of the right hand side means replacing the approximate $(0.01 S(t))$ by the true total surface renovated in $\tau$ for economic reasons.

\subsection{Demolition rates}

The demolition rate $\gamma_{i, D, e_{i}}(t)$ in the stock $S_{i, D, e_{i}}(t)$ remains to be calculated. We regard the demolition rate $\gamma$ in the whole stock $S(t)$ as time-independent: $\gamma$ is equal to the demolition

\footnotetext{
${ }^{11}$ The asymptotic values are $0 \%$ and $100 \%$.

${ }^{12}$ For the reasons already set out in the paragraph 'Climate change' of Subsection 3.1.

${ }^{13}$ The asymptotic values are $0.85 \%$ (in the year 0 the value of $p$ was $1 \%$ in Luxembourg) and $3.15 \%$ (newer versions of Giraudet et al. (2012) use the value $p=3 \%$ constantly, from the initial to the final year).
} 
rate $0.35 \%$ which was observed in $S(0)$. The calculation of $\gamma_{i, D, e_{i}}(t)$ is based on the suggestion of Sartori et al. (2009) to first demolish the low energy classes. The total destruction in $t$ in the category $D, e$ is

$$
\operatorname{Tot}_{D, e}(t)=0.0035 \times S_{D, e}(t)=0.0035 \times \sum_{i} S_{i, D, e}(t) .
$$

We model the suggestion to begin demolishing this surface in the worst energy class: if in the category $D, e$ the percentage $\frac{S_{I, D, e}(t)}{S_{I, D, e}(0)}$ of class $I$ dwellings in the year 0 that do still exist in the year $t$, is still high (already low) we demolish much (we do not demolish much) of the total

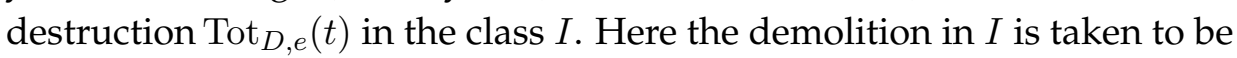

$$
\mathrm{D}_{I, D, e}(t)=\operatorname{Tot}_{D, e}(t) \times \frac{S_{I, D, e}(t)}{S_{I, D, e}(0)} \cdot 14
$$

The remainder of the total destruction is demolished in the next class:

$$
\mathrm{D}_{H, D, e}(t)=\operatorname{Tot}_{D, e}(t)-\mathrm{D}_{I, D, e}(t) .
$$

If in some year the class $I$ has been completely destroyed we destroy first in $H$, then in $G$ etc. Equation (23) and (24) can thus be written, respectively, as

$$
\mathrm{D}_{I, D, e}(t)=\frac{\operatorname{Tot}_{D, e}(t)}{S_{I, D, e}(0)} S_{I, D, e}(t)=\gamma_{I, D, e}(t) S_{I, D, e}(t),
$$

and

$$
\mathrm{D}_{H, D, e}(t)=\frac{\operatorname{Tot}_{D, e}(t)-\mathrm{D}_{I, D, e}(t)}{S_{H, D, e}(t)} S_{H, D, e}(t)=\gamma_{H, D, e}(t) S_{H, D, e}(t) .
$$

Equation (25) and (26) allow one to calculate $\gamma_{I, D, e}(t)$ and $\gamma_{H, D, e}(t)$.

\section{Description of the LuxHEI model (III)-Dynamics of the new build- ing stock}

In Section 3 we looked at the building stock which existed in 2013 (EBS), its transformations and demolitions: we calculated the evolution over time of the surface $S_{i, D, e_{i}}$ of the EBS in all $9 \times 4 \times 8$ categories $i, D, e_{i}$.

Hereinafter we study the building stock growth or new building stock (NBS). We will calculate for all $4 \times 4 \times 8$ categories $j, D, e_{j}$ the temporal development of the surface $\mathcal{S}_{j, D, e_{j}}$ (or the number $\mathcal{H}_{j, D, e_{j}}$ ) of new houses constructed in 2014 or later (in the case of new buildings $j \in\{B, A, \mathrm{ZEB}, \mathrm{PEB}\})$.

The total housing needs

$$
H=\frac{L}{\mathrm{LPH}}
$$

are the quotient of the 'population' and the 'average population per house' (if $L=500,000$ and $\mathrm{LPH}=4$ the total housing needs are 125,000). The evolution over time of $L$ is obtained exogenously using the findings of Haas and Peltier (2017). The data of STATEC (2011) suggest

\footnotetext{
${ }^{14}$ As we cannot demolish more in the class $I$ than available, the real destruction in $I$ is the minimum of $\mathrm{D}_{I, D, e}(t)$ and $S_{I, D, e}(t)$.
} 
that the number LPH of people per house decreases over time. In the model, the decrease of LPH is bounded by a minimal number ${ }^{15}$ and is calculated endogenously.

We denote the number of new constructions in 2014, 2015, etc., up to $\tau(\tau=t+1, t \geq 0)$ by $\mathcal{H}(\tau)$. The difference $(\Delta \mathcal{H})(\tau)=\mathcal{H}(\tau)-\mathcal{H}(t)$ is the number of new constructions in the year $\tau$. This number is also the difference

$$
(\Delta \mathcal{H})(\tau)=H(\tau)-\left(\frac{1}{\mathrm{SPH}} \sum_{i, D, e_{i}} S_{i, D, e_{i}}(\tau)+\mathcal{H}(t)\right)
$$

between the housing needs $H(\tau)$ in $\tau$ and the sum of the number of dwellings from 2013 and earlier that do still exist in $\tau$ and the number of new dwellings constructed in 2014, 2015, up to $t$. The existing stock $\sum_{i, D, e_{i}} S_{i, D, e_{i}}(\tau)$ is expressed in $m^{2}$ and must therefore be divided by the average surface SPH of a house which existed in 2013; because equation (27) is expressed in (number of) houses.

The surface $(\Delta \mathcal{S})(\tau)$ which corresponds to the new constructions $(\Delta \mathcal{H})(\tau)$ depends on the surface per house:

$$
(\Delta \mathcal{S})(\tau)=\operatorname{SPH}(Y(\tau))(\Delta \mathcal{H})(\tau)
$$

The surface $\operatorname{SPH}(Y(\tau))$ is an increasing function of the disposable income per capita. The value of $Y(\tau)$ for the years $\tau$ up to 2060 comes from the projections of Haas and Peltier (2017).

We model SPH $(\operatorname{SPH}(Y(\tau)))$ incorporating a maximal surface per house and the expectation that the annual increase of SPH shrinks as the surface gets closer to this limit. The modelling of the evolution over time of LPH is very similar. Base on the data of STATEC (2017) it can be observed that the surface $\Sigma=$ SPH increases by $20 \%{ }^{16}$ if the income doubles, thus

$$
\Sigma_{\tau}=\Sigma_{t}\left(1+\frac{\Delta Y}{Y} \times 20 \%\right)
$$

Over time, equation (28) produces an increasingly higher surface although $\Sigma$ is in fact bounded by a limit or maximal surface $\Sigma_{\max }$. The quotient $\frac{\Sigma_{\max }-\Sigma_{t}}{\Sigma_{\max }-\Sigma_{0}}$ allows to model the idea that $\Sigma$ increases by lower percentages than $20 \%$ when it comes closer to $\Sigma_{\max }$. Indeed, it is enough to replace (28) by equation

$$
\operatorname{SPH}(Y(\tau))=\Sigma_{\tau}=\Sigma_{t}\left(1+\frac{\Delta Y}{Y} \times \frac{\Sigma_{\max }-\Sigma_{t}}{\Sigma_{\max }-\Sigma_{0}} \times 20 \%\right)
$$

In the same way as for the EBS $S(\tau)$, we distribute the NBS $\mathcal{S}(\tau)$ amongst the categories $j, D, e_{j}$. The surface of new constructions in the category $j, e_{j}$ is

$$
(\Delta \mathcal{S})_{j, e_{j}}(\tau)=\operatorname{PRN}_{j, e_{j}}(\tau)(\Delta \mathcal{S})(\tau)
$$

The proportion $\operatorname{PRN}_{j, e_{j}}(\tau)$ of new constructions in $j, e_{j}$ is calculated exactly as the proportion in (6) and the one in (20):

$$
\operatorname{PRN}_{j, e_{j}}(\tau)=\frac{\operatorname{LCCN}_{j, e_{j}}^{-\nu(\tau)}(\tau)}{\sum_{k, e_{k}} \operatorname{LCCN}_{k, e_{k}}^{-\nu(\tau)}(\tau)}
$$

\footnotetext{
${ }^{15}$ Which is set equal to 2 .

${ }^{16}$ The percentage $20 \%$ is only valid in the categories $\mathrm{O}-\mathrm{H}$ and $\mathrm{T}-\mathrm{H}$; for $\mathrm{O}-\mathrm{F}$ and T-F it is only $1 \%$.
} 
and

$$
\operatorname{LCCN}_{j, e_{j}}(\tau)=\operatorname{INVCN}_{j, e_{j}}(\tau)+\operatorname{ENERCN}_{j, e_{j}}(\tau)+\operatorname{ICN}_{j, e_{j}}(\tau) \cdot{ }^{17}
$$

The carrier $e_{j}$ is $\mathrm{E}+\mathrm{s}$ for $j \in\{\mathrm{ZEB}, \mathrm{PEB}\}$ (see Table 3). Indeed a ZEB (PEB) is a house with a neutral (positive) annual energy balance: a ZEB (PEB) produces as much (more) energy as the household consumes over a year. This is achieved by perfect insulation and an efficient heating system. We assume households living in such buildings to have high environmental awareness; they desire sustainable heating and want to maximise the energy production from renewable energies. The model therefore only allows solar thermal heating combined with a heat pump that works mainly with electricity from the in-house photovoltaic system.

Unlike the proportions (6) and (20), the share $\operatorname{PRN}_{j, e_{j}}(\tau)$ does not dependent on the category $D$. Indeed the dependence on $D$ comes in (6) and (20) from the different discount rates used for the different categories $D$, and these discount rates are not needed in the case of new dwellings. We get the searched surface $(\Delta \mathcal{S})_{j, D, e_{j}}(\tau)$ by the formula

$$
(\Delta \mathcal{S})_{j, D, e_{j}}(\tau)=\operatorname{PR}_{D}(\tau) \operatorname{PRN}_{j, e_{j}}(\tau)(\Delta \mathcal{S})(\tau)
$$

where $\mathrm{PR}_{D}(\tau)$ is the proportion of $D$-dwellings (owner-occupied houses when $D=\mathrm{O}-\mathrm{H}$ ) in the new constructions in the year $\tau$. As $D=\mathcal{P} \cap \mathcal{T}(\mathcal{P} \in\{\mathrm{O}, \mathrm{T}\}, \mathcal{T} \in\{\mathrm{H}, \mathrm{F}\})$, we have

$$
\operatorname{PR}_{D}(\tau)=\operatorname{PR}_{\mathcal{T}}(\tau) \operatorname{PR}_{\mathcal{P} \mid \mathcal{T}}(\tau)
$$

The percentage $\operatorname{PR}_{\mathcal{P} \mid \mathcal{T}}(\tau)$ was observed in the year $\tau=1$ and we use that value. As concerns the two shares $\operatorname{PR}_{\mathcal{T}}(\tau)$, they are known once we found the percentage $\operatorname{PR}_{\mathrm{F}}(\tau)$ of flats in the new constructions in $\tau$. Notice that we take into account that the latter percentage increases over time. A complete description of this modelling method can be found in Appendix A.4.

Table 3 - Initial construction costs $\operatorname{INVCN}_{j, e_{j}}(0)$ (in $€ / m^{2}$ )

\begin{tabular}{|r|c|c|c|c|}
\cline { 2 - 5 } \multicolumn{1}{c|}{} & B & A & ZEB & PEB \\
\hline Oil & 2527 & 2716 & & \\
\hline Gas & 2504 & 2692 & & \\
\hline Electricity & 2582 & 2776 & & \\
\hline Pellets & 2588 & 2782 & & \\
\hline Oil+Solar & 2626 & 2823 & & \\
\hline Gas+Solar & 2603 & 2799 & & \\
\hline Electricity+Solar & 2682 & 2883 & 3084 & 3285 \\
\hline Pellets+Solar & 2688 & 2889 & & \\
\hline
\end{tabular}

\section{Description of the energy policy tools}

With the building sector being accountable for about half of the EU's energy needs and greenhouse gas (GHG) emissions (Lechtenböhmer and Schüring, 2011), the sector is crucial to meet large energy and climate objectives (for example, the EU's 20-20-20 strategy or the Paris Agreement), and so is the residential building sector (Itard, 2008). Also in the Luxembourgish energy

\footnotetext{
${ }^{17}$ The initial construction costs $\operatorname{INVCN}_{j, e_{j}}(0)$ were again determined after concertation with experts from renovation companies and do only contain direct building costs, that is, no land costs are included.
} 
policy the building sector is of particular importance: since the 1990s the government promotes energy conservation in the building stock through the application of various policy instruments. More precisely, energy policy tools of direct nature (regulatory instruments) and indirect nature (communication or financial instruments) were implemented to address the barriers that hinder the exploitation of the sector's large energy conservation potential (often referred to as the energy efficiency gap or paradox). In the LuxHEI model we evaluate the effects of currently applied and possible future financial and regulatory instruments. A detailed synopsis of the instruments and their modelling is provided in this section.

\subsection{Existing instruments-initial and extended form}

\subsubsection{Capital grants}

Since 2013 the Luxembourgish state offers PRIMe-House capital grants to promote household investments in insulation measures on existing dwellings, green building and sustainable heating systems. ${ }^{18}$

The subsidy $\left(\mathcal{S}_{1}\right)$ granted for insulation measures in an existing dwelling increases with the quantity of insulation material used (including windows) and with its quality. Since information about material properties is not captured by the model, we use available data of Myenergy Luxemgourg (2018) ${ }^{19}$ to compute that for a retrofit from $i$ to $f, 15 \%$ of the average capital expenditures for insulation measures are covered by this policy tool (up to 2026). ${ }^{20}$

Between 2013 and 2016, state aids were also granted for all new energy class $B$ and $A$ constructions (for example, the maximal grant for a new single-family house of class $A$ was $24,000 €$ in 2014). With the introduction of the Luxembourgish environmental certification system LENOZ in 2016, the regulations of this policy changed. The new scheme determines a building's sustainability no longer exclusively by its energy class but also through its geographical location and factors of economic and social nature. To benefit from the grant, new constructions must now obtain a certain amount of points in the LENOZ-evaluation. Since such specifications are not tangible for the model, we build on the assertions of consultants from Myenergy and assume that between the beginning of 2017 and the end of 2020, 35\% of new energy class $A$ dwellings, $50 \%$ of new ZEB, and 65\% of new PEB remain eligible for this second type of PRIMe-House grant $\left(\mathcal{S}_{2}\right)$.

Until the end of 2024, the Luxembourgish state also offers grants for solar thermal plants, pellet heating systems and heat pumps (varying between 2,500€ and 8,000€). An additional subsidy (of 1,000€) is accorded whenever the two latter systems are combined with a solar thermal plant. As the overall costs of a heating system replacement are split into system and installation costs (included in the investment costs $\operatorname{INVC}_{i, f}(\tau)$ ) and ancillary costs induced by the carrier swap (included in the switching costs $\left.\mathrm{SWIC}_{e_{i}, e_{f}}\right)$, this last type of grant $\left(\mathcal{S}_{3}\right)$ is split $\left(\mathcal{S}_{3}=\mathcal{S}_{3}^{a}+\mathcal{S}_{3}^{b}\right.$, $\mathcal{S}_{3}^{a}$ and $\mathcal{S}_{3}^{b}$ are fixed percentages of $\mathcal{S}_{3}$ ):

$$
\begin{gathered}
\operatorname{INVC}_{i, f}(\tau)^{\prime}=\operatorname{INVC}_{i, f}(\tau)-\left(\mathcal{S}_{1}(\tau)+\mathcal{S}_{3}^{a}(\tau)\right), \\
\operatorname{SWIC}_{e_{i}, e_{f}}(\tau)^{\prime}=\operatorname{SWIC}_{e_{i}, e_{f}}-\mathcal{S}_{3}^{b}(\tau),
\end{gathered}
$$

\footnotetext{
${ }^{18}$ Instruments that were already applied in 2013 are considered in the calibrations of the model.

${ }^{19}$ Myenergy is the main national structure to promote the transition to sustainable energy.

${ }^{20}$ Note that this modelling choice reflects reality as the level of subsidies increases, as it should, with the quality of the final energy class $f$, that is, the number or grade of the undertaken actions.
} 


$$
\operatorname{INVCN}_{j, e_{j}}(\tau)^{\prime}=\operatorname{INVCN}_{j, e_{j}}(\tau)-\left(\mathcal{S}_{2}(\tau)+\mathcal{S}_{3}(\tau)\right) .
$$

Based on the findings of ADEME (2013), we take into account that probably not all eligible households actually use the capital grants; mainly because of imperfect information. Due to the substantial efforts of the Luxembourgish government to promote the PRIMe-House grants, we assume, however, that the utilisation rates are slightly higher than those in France (ADEME, 2013): in Luxembourg, on average, $75 \%$ of retrofits and $90 \%$ of new constructions apply the instrument.

In addition, an extended version of the policy is modelled. This means that at the end of the instruments' initial application period, the model prolongs the grants for 15 additional years and considers their application as mandatory; as in Giraudet et al. $(2011,2012)$ all eligible households apply the instrument.

\subsubsection{Subsidised loans}

With the launch of the Luxembourgish climate bank in 2017, households became eligible for a retrofitting credit at reduced interest or even zero-interest rate. Under the interest-free loan, recipients can take out a credit of up to 50,000€, repayable (without interests) within 15 years, and further get a capital grant of $10 \%$ of the loan. On the contrary, under the loan at reduced interest rate, the credit is limited to a maximum of $100,000 €$, repayable within 15 years, and the state grants a $1.5 \%$ subsidy on the interest rate of the bank.

To encode both retrofitting credits, we again refer to ADEME (2013) and consider that not all households borrow money to pay a retrofit. Similarly to the situation observed in France, we assume the proportion $\mathcal{P}$ of retrofitting households taking out a loan to be $30 \%$, whether or not the policy tool is applied. For this proportion $\mathcal{P}$, the investment $\operatorname{costs} \operatorname{INVC}_{i, f}(\tau)$ are then increased by the accrued interests under an averaged fixed interest rate ${ }^{21}$. Only if the interest-free or reduced interest loan is applied by the government, the increased investment costs $\operatorname{INVC}_{i, f}(\tau)$ of the proportion $\mathcal{P}$ are decreased by the saved interests. Furthermore, the instruments' effectiveness is improved by limiting their duration of application (Köppel and Ürge-Vorsatz, 2007): as for capital grants, the subsidised loans are available until 2026.

Comparably with the extended version of the PRIMe-House grants, we encode a complementary scenario where both instruments' period of application is prolonged until 2041 (+15 years), and where for the reduced interest loan the $1.5 \%$ subsidy is increased to $2.0 \%$.

\subsubsection{Energy tax}

Since the European Commission implemented the EU Energy Taxation Directive (ETD) (Matteoli, 2003) in 2003, minimum tax rates are imposed on energy products in the Member States. Within this framework, the Luxembourgish government taxes the use of electricity and fuels like oil, gas and coal (if they are not used to produce electricity). In Luxembourg, the tax rates depend on the energy source (the carrier), their sector of application and the volume of the annual consumption. Based on the data of the Ministry of the Economy of Luxembourg (2017) we find that carriers used for space heating in the residential sector are taxed between $1.5 € / \mathrm{t} \mathrm{CO}_{2}$ and $5 € / \mathrm{t} \mathrm{CO}_{2}$. In this first form of the policy tool we consider the energy tax as time-independent

\footnotetext{
${ }^{21}$ This rate corresponds to the mean of the fixed rates that Luxembourgish banks charged on mortgage loans between 2009 and 2017.
} 
and encode the instrument by adding the amount of the tax (converted into $€$ per $\mathrm{kWh}$ ) to the energy price $P_{e}(t)$ of the corresponding carrier $e$.

An enhanced energy tax is also included in our model: following the objective of the Ministry of the Economy of Luxembourg et al. (2017) to raise the taxes on energy products, we increase the initial level of taxation by $100 \%$ every 10 years. The first increase is implemented in 2025 , the last in 2055.

\subsubsection{Energy performance requirements for new buildings}

In Luxembourg, the energy efficiency of new residential buildings is prescribed by law since November 2007. This building code dictated that as of 1 January 2008, all new constructions needed to have at least the energy efficiency energy class $D$. The standard was then increased to energy class $B(A)$ in 1 July 2012 (1 January 2015). This is why in the proportion $\operatorname{PRN}_{j, e_{j}}(\tau)$ of new constructions, $j \in\{B, A, \mathrm{ZEB}, \mathrm{PEB}\}$ in $2014(j \in\{A, \mathrm{ZEB}, \mathrm{PEB}\}$ as of 2015).

Once again, an extra scenario in which building codes are further tightened is included: as of 1 January 2030 (2045) the standard ZEB (PEB) becomes mandatory for new constructions.

\subsection{Possible future instruments}

\subsubsection{Remediation duty for existing buildings}

As most Member States, Luxembourg does not specify minimum energy efficiency standards for existing residential buildings (BBSR, 2016). The only obligation with regard to existing dwellings is to respect minimum material standards when retrofitting. However, in Luxembourg's neighbouring countries (Germany and France), stricter requirements on the existing building stock do exist. In Germany, partial renovation is for example mandatory after the acquisition of an existing building: if a dwelling was bought or inherited after 1 February 2002 the new owners must either insulate the roof or the top floor ceiling. As regards France, the National Assembly adopted on 26 May 2015 a bill which stipulates that every dwelling must be retrofitted until 2025 if its overall primary energy consumption is above $330 \mathrm{kWh} / \mathrm{m}^{2} /$ year; in the LuxHEI model this corresponds to a house of energy efficiency class $G$. The bill also dictates that as of 2030, all dwellings must be retrofitted before they can be listed for rent or sale.

In light of the energy saving and $\mathrm{CO}_{2}$ mitigation potential of the existing building stock (Petersdorff et al., 2006; Tommerup and Svendsen, 2006) and the rules deployed in two of Luxembourg's three bordering countries, we included a remediation duty in the LuxHEI model: as of 2022 (considered as the closest possible year for implementation), all residential buildings that are listed for rent or sale must be retrofitted to an overall primary energy class above $H$. To ensure effectiveness of this tool, the regulation is tightened by one energy class every five years: buildings whose inhabitants switch must at least reach class $F$ as of 2027, $E$ as of 2032, and $D(C)$ as of 2037 (2042).

To model this policy, we begin by considering that without remediation duty, a fraction $\zeta$ of the proportion $\mathrm{X}_{i, D, e_{i}}(\tau)$ of retrofits of dwellings of class $i$ is induced by an inhabitant switch. On the contrary, whenever the instrument is applied, all inhabitant switches are followed by a retrofit. To avoid double counting when the remediation duty is in force, the fraction $\zeta \mathrm{X}_{i, D, e_{i}}(\tau)$ must be subtracted from $\mathrm{X}_{i, D, e_{i}}(\tau)$, and equation (5) of the model has to be changed to:

$$
\operatorname{TRANS}_{i, f ; D, e_{i}}(\tau)=\left(1-\gamma_{i, D, e_{i}}(t)\right) S_{i, D, e_{i}}(t)\left(Z_{i, D, e_{i}}(\tau)+\left(\mathrm{X}_{i, D, e_{i}}(\tau)(1-\zeta)\right)\right) \operatorname{PR}_{i, f} ; D, e_{i}(\tau),
$$


for all $i<i_{\min }$ and all $f \geq i_{\min }$. The percentage $Z_{i, D, e_{i}}(\tau)^{22}$ is the proportion of owner-occupied or rented dwellings that change occupancy in the year $\tau$, and the class $i_{\min }$ is the required lowest efficiency class. More specifically, if $i<i_{\min }$ and $f<i_{\min }$, we set $\mathrm{PR}_{i, f} ; D, e_{i}(\tau)=0$, and if $i \geq i_{\min }$, then $f>i_{\min }$ and in this case we do not change the original formula.

\subsubsection{Carbon tax}

In 2005, the world's first and largest international emissions trading system, the European Union Emissions Trading System (EU ETS), was implemented to reduce greenhouse gas (GHG) emissions in the EU. This system, however, covers only about $45 \%$ of the EU's GHG emissions since it does not cap the volume of gases emitted by the agriculture, residential and transportation sectors. For these three sectors, binding national targets are fixed through the Effort Sharing Decision (ESD).

To meet these targets in a cost-effective way, a carbon tax is often recommended by environmental economists (Pearce, 1991; Gerlagh and Van der Zwaan, 2006; Ghalwash, 2007). As several attempts to introduce an EU-wide carbon tax failed, we consider in our model a national carbon tax that applies a uniform price to emissions from all sources and sectors (Bruvoll and Larsen, 2004; Lin and Li, 2011). For this purpose we set the initial level of the tax equal to the price of the EU ETS allowances (Weisbach, 2012), and increase the level over time to enhance the reduction of $\mathrm{CO}_{2}$ emissions progressively (Peck and Teisberg, 1992). More precisely, the tax is based on the predicted annual price increase of EU ETS certificates (Capros et al., 2016). The carbon tax (in the residential sector) will thus increase from $15 € / \mathrm{tCO}_{2}$ in the starting year 2020 of the policy tool to $33 € / \mathrm{tCO}_{2}$ in 2030 and $89 € / \mathrm{tCO}_{2}$ in 2050. In the second half of the century, carbon emissions are projected to decrease (Chakravorty et al., 1997; OECD et al., 2015) and the level of the tax is estimated to decline (Vollebergh, 2014). We therefore assume that the carbon tax comes down to $80 € / \mathrm{tCO}_{2}\left(72 € / \mathrm{tCO}_{2}\right)$ in 2055 (2060).

The modelling of the carbon tax is similar to the encoding of the energy tax: the price of the tax (in $€$ per $\mathrm{kWh}$ ) is added to the energy price $P_{e}(t)$ of the corresponding carrier $e$.

\section{Results and discussion}

We now evaluate the policy tools of Section 5 in a similar manner as in Amstalden et al. (2007); Köppel and Ürge-Vorsatz (2007); McCormick and Neij (2009); Giraudet et al. (2011); Knobloch et al. (2019). For this purpose, different model scenarios are generated: firstly the model is run without any instrument (this baseline scenario serves as a benchmark for the following evaluation), secondly each instrument is put in force individually (the original and extended forms of the existing policy tools are examined), thirdly various bundles of instruments are studied (the bundle of all existing initial tools, the bundle of all existing extended tools and the bundle of all existing and possible future tools). After each run, the scenario is assessed with regard to its environmental and economic effectiveness, and its potential to help achieving the Luxembourgish energy and emission targets is determined. Actually, in order to contribute to the EU's 20-20-20 strategy, Luxembourg must decrease by $20 \%$ its final energy consumption (in comparison with the 2007 level) as well as its $\mathrm{CO}_{2}$ emissions from sectors outside the EU ETS (in comparison with the 2005 levels). In the period 2021-2030, emission cuts of even 40\% must be achieved (relative to the 2005 levels). Although the national targets are not limited to

\footnotetext{
${ }^{22}$ The data of Eurostat (2017) shows that $Z_{i, D, e_{i}}(\tau)$ is $1.3 \%(6 \%)$ for owner-occupied (rented) dwellings.
} 
a single sector, the second part of our assessment is interesting since the building sector offers one of the greatest potentials to decrease energy consumption and $\mathrm{CO}_{2}$ emissions (Schulz, M., and Mavroyiannis, A. D., 2012; TIR Consulting Group LLC and Grand Duchy of Luxembourg Working Group, 2016), and this at comparatively low costs (Levine et al., 2007; Schimschar et al., 2011).

A detailed overview of the parameters that were not yet specified but used in the model-runs, can be found in the table below.

Table 4 - Parameter overview

\begin{tabular}{|c|c|c|c|}
\hline Parameter & Signification & Setting & Literary basis \\
\hline$\alpha$ & $\begin{array}{l}\text { percentage of the } \\
\text { initial retrofitting } \\
\text { costs that cannot } \\
\text { be decreased by } \\
\text { experience }\end{array}$ & $20 \%$ & Giraudet et al. (2012) \\
\hline$l$ & learning-by-doing rate & $10 \%$ & $\begin{array}{l}\text { Weiss et al. (2010); Gi- } \\
\text { raudet et al. (2012) }\end{array}$ \\
\hline$N$ & $\begin{array}{l}\text { average lifetime of a } \\
\text { retrofit }\end{array}$ & 35 years & $\begin{array}{l}\text { Ministry of the Econ- } \\
\text { omy of Luxembourg } \\
\text { and Lichtmeß (2014) }\end{array}$ \\
\hline $\mathcal{G}$ & green value & $33 \%$ & Högberg (2013) \\
\hline$\beta$ & $\begin{array}{l}\text { constant percentage of } \\
\text { the initial intangible } \\
\text { costs }\end{array}$ & $20 \%$ & Giraudet et al. (2012) \\
\hline$\mu$ & $\begin{array}{l}\text { information accelera- } \\
\text { tion rate }\end{array}$ & $25 \%$ & Giraudet et al. (2012) \\
\hline$M$ & $\begin{array}{l}\text { average lifetime of a } \\
\text { carrier switch }\end{array}$ & $20 \%$ & $\begin{array}{l}\text { Ministry of the Econ- } \\
\text { omy of Luxembourg } \\
\text { and Lichtmeß (2014) }\end{array}$ \\
\hline
\end{tabular}

\subsection{Baseline projection}

The model projects for 2060 a total final energy consumption of 4,122 GWh ( $-9 \%$ compared to 2014) and total $\mathrm{CO}_{2}$ emissions of $734,600 \mathrm{tCO}_{2}$ (-20\% compared to 2014) (see Table 5 and Figure 3). The major growth of the residential building stock (Figure 1) is due to the projected increase of the Luxembourgish population: based on the 3\% GDP growth scenario of Haas and Peltier (2017) and the baseline population projections of Eurostat, we can assert that Luxembourg will face the largest population growth rate (approximately 98\% between 2014 and 2060) among all EU Member States. At the end of the model's projection period, the (cumulated) new building stock (NBS) therefore corresponds to $66 \%$ of the total building stock. On this basis, savings in energy consumption and $\mathrm{CO}_{2}$ emissions can only be achieved through changes in the sector's energy efficiency or energy sufficiency. As concerns the gains in energy efficiency, they are mainly realised by a transformation of the existing building stock (EBS), that is, demolitions and retrofits, and the construction of a highly efficient NBS. More precisely, in 2060, the final energy consumption per square meter has fallen by $61 \%$ (in comparison with 2014) (Table 5), $69 \%$ of the total dwellings have at least the energy class $B$ (compared to $7 \%$ in 2014), and $32 \%$ of the households make use of solar thermal energy to support their heating system (Table 7, Figures 5 and 6). Such large energy efficiency increases are, however, followed by a significant rebound effect: by the end of the projection period the adjustment factor raised by 38\% (Table 6 and Figure 2). This is due to the fact that all along the modelling period, the continuously increasing 
share of dwellings with a low $\rho_{k, e}$ compensates the natural raise of the energy price $P_{e}(t)$, thus generating a shrinking of the adjustment factor's independent variable $\rho_{k, e} P_{e}(t)$ and hence a higher overall factor $F(t)$. Concerning the country's energy objectives, none of them can be achieved in the residential building sector (Table 9): the 2020 levels of final energy consumption $\left(\mathrm{CO}_{2}\right.$ emissions) are 29\% (7\%) higher than the 2007 levels (2005 levels), and the 2030 emissions are projected to decrease only by $4 \%$ instead of $40 \%$ (compared to 2005).

Notice that ignoring the effects of climate change, the green value, and the dynamic evolution of the new constructions' building type, changes the outcomes of the baseline scenario for the worse. Actually, compared to the baseline projection that contains all new features (see above), we end up with a total final energy consumption that is $8 \%$ higher $(4,478 \mathrm{GWh})$ and total $\mathrm{CO}_{2}$ emissions that are increased by $10 \%\left(805,600 \mathrm{tCO}_{2}\right)$. This is inter alia due to the fact that at the end of the projection period, the total number of retrofits has decreased by $50 \%$ [compared to the projection with all new features], only $0.2 \%(0.0 \%)$ of the total building stock correspond to ZEB (PEB) [compared to $0.8 \%(0.5 \%)$ ], and just $27 \%$ make use of solar thermal energy to support their heating system [compared to $32 \%$ ].

\subsection{Evaluation of the individual energy policy tools}

In the following evaluation, the effects of the 10 single-instrument scenarios (see above) are compared to the baseline scenario. The ranking 1-10 means 'most effective-least effective'; the description of the results starts at 'rank 10' and ends with 'rank 1'.

\subsubsection{Places 10 to 7: Subsidy schemes}

No significant variations (about 1\% at most) from the baseline are observed in the initial and extended forms of the subsidised loan and capital grant scenarios.

From an ecological and economic viewpoint, the initial subsidised loan scenario is the least effective. Compared to the baseline projection virtually no reduction effects can be observed: additional energy and emission savings of $0.01 \%$ (Table 5, Figures 3 and 4 ) are realised, for a benefit to cost ratio ${ }^{23}$ of $101 €$ per $\mathrm{kWh}$ saved (Table 8).

In the enhanced version of the subsidised loan scenario, whilst energy and emission savings increase slightly $(-0.02 \%$; Table 5 , Figures 3 and 4$)$, cost-effectiveness deteriorates $(+26 \%$; Table 9).

A bit better results are achieved in the scenario with the initial form of the capital grants: energy savings $(-0.17 \%)$ and emission reductions $(-0.47 \%)$ can be observed in 2060 (Table 5), for a benefit to cost ratio of $74 €$ per $\mathrm{kWh}$ saved (Table 8 ). Although the instrument induces gains in energy efficiency ( $-0.21 \%$ of conventional energy consumption in 2060), a decrease of the adjustment factor is observed $(-0.05 \%$ in 2060) (Table 6 and Figure 2$)$. This small prebound effect is mainly due to the somewhat greater use of electricity, an energy carrier with a comparatively high energy price. Compared to the baseline scenario, a small decrease in accumulated retrofits is also observed at the end of the projection period ( $-0.05 \%$; Table 6 and Figure 7). This is due to the fact that capital grants boost the number of retrofits during their application period so that less lucrative retrofitting options remain after that period.

\footnotetext{
${ }^{23}$ The benefit to cost ratio corresponds to the difference of the 2060 final energy consumption of the baseline scenario and the 2060 final energy consumption of the corresponding policy tool scenario, divided by the financial incentives accumulated during the policy's application period.
} 
Although the environmental effectiveness of capital grants more than doubles in the enhanced scenario (Table 5), its cost-effectiveness changes for the worse $(+40 \%$; Table 8$)$.

\subsubsection{Places 6 and 5: Energy taxes}

Next ranks the initial [extended] form of the energy tax scenario: state revenues of 14 [8] $€$ per $\mathrm{kWh}$ saved (Table 8) come along with a decreased total final energy consumption $(-0.34 \%$ $[-1.61 \%])$ and decreased total $\mathrm{CO}_{2}$ emissions (-0.37\% [-1.77\%]) (Table 5, Figures 3 and 4$)$ in 2060. On the contrary to the initial and extended forms of the capital grant scenario, the savings come rather from a better energy sufficiency (adjustment factor in 2060: $-0.17 \%$ $[-0.81 \%]$ ) than from gains in energy efficiency (conventional energy consumption in 2060: $-0.02 \%[-0.08 \%]$ ) (Table 6). The prebound effect induced by the increased price $P_{e}(t)$ of most carriers $e$ [largely] offsets the rebound effect caused by the slightly more efficient building stock. At the end of the projection, no substantial deviation from the baseline can be observed in the total number of retrofitted dwellings and in the performance of the total final building stock (Tables 6 and 7).

\subsubsection{Places 4 and 3: Remediation duty and carbon tax}

Significant savings are reached under the remediation duty and the carbon tax. Compared to the baseline, the remediation duty [carbon tax] reduces the final energy consumption by $4.58 \%$ [5.38\%] and the carbon dioxide emissions by 5.28\% [6.04\%] (Table 5, Figures 3 and 4). Although both possible future instruments result in comparable savings, the way in which they are achieved is different.

Among all 10 single-instrument scenarios, the remediation duty generates naturally the largest increase in retrofitted buildings ( $+39 \%$ in 2060; Table 6 and Figure 7). From Table 6 we get that this not only induces the second-highest increase in energy efficiency (conventional energy consumption in 2060: $-8.01 \%$ ) but also the second-highest rebound effect (adjustment factor in 2060: $+1.17 \%$ ). We observe in Figure 7 that each tightening of the remediation duty causes a prompt raise in annual retrofits. However, after the last tightening of the regulation, the prompt raise in retrofits is followed by a steady drop. Similarly to the phenomenon observed in the capital grant scenarios, annual demolitions and retrofits shrink the number of buildings that are affected by the remediation duty. Relative to the baseline, a visibly higher share of solar energy is achieved by the policy tool, and while it decreases the share of energy efficiency classes below $E$, an increase in the share of the classes $E, D$ and $C$ is observed (Table 7, Figures 5 and 6). From a governmental perspective the scenario generates no direct expenses or revenues (Table 8).

In contrast to this but comparable to the effects already observed in the energy tax scenarios, the carbon tax generates state revenues of $10 €$ per kWh saved (Table 8) and realises its savings (conventional energy consumption in 2060: $-0.35 \%$ ) less through performance improvements but rather through a more conscious heating behaviour; implying the strongest decrease of the adjustment factor (-3.00\% in 2060) (Table 6). Furthermore, no significant variations from the baseline are observed in the quantity of retrofits, as well as in the share of energy classes and carriers in the total final building stock (Table 6, Figures 5, 6 and 7). 


\subsubsection{Places 2 and 1: Performance requirements}

In the initial form of the scenario with energy performance requirements for new buildings, energy conservation (total final energy consumption in 2060: $-2.06 \%$ ) is below the savings of the remediation duty and the carbon tax scenarios (Table 5 and Figure 3). The scenario's emission mitigation (total $\mathrm{CO}_{2}$ emissions in 2060:-10.04\%) are, however, well above those of the two previous scenarios, so that the mean value of energy and emissions savings becomes the second-highest among all 10 single-instrument scenarios (Table 5 and Figure 4). In this case, energy conservation is achieved through the joint decrease of the 2060 conventional energy consumption $(-0.86 \%)$ and the adjustment factor $(-2.03 \%)$ (Table 6$)$. The regulation implies that as of 2015 only 3 performance classes are allowed for new buildings ( $A$, ZEB and PEB); a large majority goes for energy efficiency class $A: 62.7 \%$ of the buildings in the total stock of 2060 have class $A$, compared to $3 \%$ with class ZEB or PEB (Table 7). In comparison with the baseline there is also a trend for more solar thermal energy $(34.2 \%$ of the total final building stock) (Table 7).

By far the best environmental-effectiveness is reached in the extended form of this policy tool: not only does the instrument realise major energy savings (final energy consumption in 2060: $-32.87 \%$ ), but also does it achieve massive carbon dioxide reductions (total $\mathrm{CO}_{2}$ emissions in 2060: $-48.62 \%$ ) (Table 5, Figures 3 and 4). These major savings particularly stem from a much more efficient NBS which induces the largest decrease of the total conventional energy consumption in 2060 (-27.53\%; Table 6). The share of dwellings in the total final stock with an energy efficiency class above $B$ is equal in the initial and extended forms of the building code, but a clearly higher share of ZEB (12.6\%) and PEB (26.7\%) exists in the latter form (Table 7, Figures 5 and 6). Note that although the NBS increases continuously, the greater construction of PEB (as of 2030) results in a strong decrease of the NBS' final energy consumption (Figure 8), induced by the new buildings' energy production. Remarkable is also that in 2060 more than half of the total building stock uses solar thermal energy to support their heating system (Table 7 and Figure 6). These large performance improvements are followed by a comparably large rebound effect: with a raise of $3.60 \%$ in 2060 , the highest increase of the adjustment factor is observed in this scenario (Table 6 and Figure 2). Moreover, no direct state revenues or expenses are generated by the policy tool (Table 8).

While none of the single-instrument scenarios achieves the country's energy and climate objectives in time, the extended form of the energy performance requirements for new buildings is the only tool that accomplishes the 2020 energy targets as well as the 2030 emission goals (not in time but) in the course of the projection period (Table 9). The latter goal ( $-40 \%$ of total $\mathrm{CO}_{2}$ emissions compared to 2005) is fulfilled in 2050, and the energy target ( $-20 \%$ of total final energy consumption compared to 2007) is realised in 2055.

\subsection{Evaluation of combined energy policy tools}

In the previous section we observed that the 10 single-policy instruments have different ecological and financial impacts. To accumulate the advantages of these instruments, policy makers normally combine the tools in packages and apply them simultaneously. In addition to the standard evaluation of the scenarios' effectiveness (see above), we now evaluate whether or not instruments generate synergistic effects when applied concurrently. Therefore 3 multipleinstrument scenarios are run: the first (second) consists of the existing instruments in their 
initial (extended) form; the third corresponds to the second-except that we consider the initial form of the energy tax and include the two possible future instruments.

\subsubsection{Bundle 1}

From an environmental viewpoint, running the model with the policy mix that is currently applied by the Luxembourgish state results in a decrease of the total final energy consumption $(-2.45 \%$ in 2060$)$ and $\mathrm{CO}_{2}$ emissions $(-10.53 \%$ in 2060$)$ that is almost identical to the cumulated decrease of the corresponding single-instrument scenarios (Table 5, Figures 3 and 4). In this first instrument package, the effects of capital grants, subsidised loans, and the energy tax are insignificant, so that the observed savings mainly stem from the performance requirements. Many outcomes of the multiple-instrument scenario are hence similar to those of the building code scenario: energy conservation comes (in the bundle, just as in the building code) from an interplay of better energy efficiency (total conventional energy consumption in 2060: $-1.05 \%$; Table 6) and energy sufficiency (adjustment factor in 2060: -2.14\%; Table 6 and Figure 2); the share of energy classes and carriers in the total final building stock is also nearly identical (in the bundle and in the building code) (Table 7, Figures 5 and 6).

From a financial viewpoint, we are interested in the (negative) balance of state revenues minus state expenses. The absolute value of this balance is greater than the cumulated balances of the individual policy tools. The state revenues of the policy package are below those of the energy tax scenario (due to a lower energy consumption) and the state expenses are greater than the cumulated expenses of the capital grant and subsidised loan scenario (due to a higher share of eligible dwellings). With a benefit to cost ratio of $5 €$ per $\mathrm{kWh}$ (Table 8 ) the scenario's higher energy savings lead to a significantly better economic effectiveness.

Apart from this, the 2020 emission target is realised during the projection period (in 2047) (Table 9).

\subsubsection{Bundle 2}

Further improvements in environmental and economic effectiveness are achieved in the second policy package scenario: compared to the 2060 baseline levels, energy savings of $34.60 \%$ and carbon dioxide reductions of 50.71\% (Table 5, Figures 3 and 4) are realised.

Environmentally, similar to Bundle 1, the savings of Bundle 2 are almost identical to the cumulated decreases of the corresponding single-instrument scenarios (Table 5, Figures 3 and 4). The presence of capital grants, subsidised loans and the energy tax in the package leads to a greater energy efficiency than in the extended building code scenario (total conventional energy consumption in 2060: $-28.13 \%$; Table 6) and to a lower rebound effect (adjustment factor in 2060: $+2.90 \%$; Table 6 and Figure 2).

Compared to Bundle 1, the balance of state revenues and expenses more than quadruples. Due to the much larger energy savings in Bundle 2, the scenario yet generates a benefit to cost ratio of $2 €$ per kWh saved (Table 8), which means that the economic effectiveness is further increased.

In Bundle 2, even though the energy and emission targets are not achieved in time, they are all achieved within the projection period (Table 9). The 2020 energy objective is realised in 2054, and the 2020 (2030) emission goal in 2037 (2044). 


\subsubsection{Bundle 3}

The highest and most cost-effective energy and emission savings are realised in the multipleinstrument scenario of Bundle 3: for state expenses of less than $1 €$ per $\mathrm{kWh}$ saved (Table 8), the 2060 final energy consumption decreases by $42.37 \%$ and the $\mathrm{CO}_{2}$ emissions by $59.53 \%$ (Table 5 , Figures 3 and 4).

Despite the very large performance improvement of the total building stock (total conventional energy consumption in 2060: $-36.32 \%$; Table 6), the scenario projects a relatively low rebound effect (adjustment factor in 2060: $+2.40 \%$; Table 6 and Figure 2). This is mostly due to the carbon tax, an instrument that realises most of its savings through a better energy sufficiency. Moreover, the higher quantity of cumulated annual retrofits in 2060 (about $+39 \%$; Table 6 and Figure 7) is due to the incorporation of the remediation duty. This instrument, together with the performance requirements for new constructions, is primarily responsible for the share of efficient energy classes and carriers in the total building stock of 2060, which is the highest among all 10 scenarios (Table 7, Figures 5 and 6).

Similarly to Bundle 2, all national energy and emission targets are reached belatedly (Table 9): while a decrease of the final energy consumption by $20 \%$ is reached in 2048 , emissions mitigation of $20 \%(30 \%)$ are realised in $2034(2040)$.

\section{Conclusion}

With a focus on the Grand Duchy of Luxembourg, the present paper evaluates the influence of energy policy tools on final energy consumption and direct $\mathrm{CO}_{2}$ emissions in the residential building sector. For this purpose, we developed the advanced version LuxHEI of the French hybrid energy-economy model Res-IRF (Giraudet et al., 2012), which we also customised to the truly specific characteristics of Luxembourg. ${ }^{24}$ The LuxHEI model is an energy policy model that is based on economic principles and that takes into account global warming, the green value, sustainable energy efficiency classes and energy carriers, a limited availability of carriers... Based on our model's results, four principal conclusions can be drawn. Firstly, we observe that building codes generate the largest energy conservation and mitigation of carbon dioxide emissions, without requiring direct government spending. Secondly, environmental effectiveness is achieved differently depending on the instrument type: while subsidy schemes and regulations mainly affect the building stock's energy efficiency, taxes usually induce a more conscious heating behaviour. Thirdly, when used simultaneously, policy tools neither counteract nor generate direct synergistic effects but their individual impacts are more or less added-up. Therefore, the policy package with the greatest number of instruments (Bundle 3) also generates the largest effects. Fourthly, in none of the evaluated policy scenarios the national energy and emission targets are achieved on time.

Although we encoded quite a few new features (for example, more sophisticated behavioural factors) to increase our dynamic simulation model's level of realism, it remains a stylised illustration of the real world. This means that modelling assumptions (for example, about households' decision-making behaviour and the evolution of the new dwellings' surface or building type) and parameterisation hypotheses (for example, about climate change or population growth) are still subject to uncertainty. Changing these suppositions might affect the

\footnotetext{
${ }^{24}$ We again wish to point out that whenever the data is sufficient to realise the above calibrations and parameterisations, then the LuxHEI model also allows to perform the present study for any other country.
} 
scenarios' outcomes to a certain extent. As concerns the barriers to energy efficiency, not all of them are fully representable in a model (especially those of behavioural nature) and the LuxHEI model's projections may therefore be somewhat optimistic. Apart from this, we did not directly encode the impact of communicative policy tools, which tend to nudge households to behave in a more environmentally conscious way. Even if the energy saving potential of such instruments is relatively small (Gillingham et al., 2018), their absence in the model may induce a bit too pessimistic results; thus counteracting the preceding limitation. We are hence confident that the model's predictions are fairly accurate.

The policy recommendations that accompany our analysis are in compliance with other studies (Schaefer et al., 2000; Köppel and Ürge-Vorsatz, 2007; Weiss et al., 2012). More specifically, in the case of Luxembourg, these policy advices can be phrased as follows. Because all instruments have their pros and cons, and induce higher overall effectiveness when applied concurrently, a suitable combination of energy policy tools is advisable for the Luxembourgish residential building sector. In this policy mix, regulatory instruments should play a central role as they have the potential to strongly decrease the sector's energy consumption and $\mathrm{CO}_{2}$ emissions at low governmental costs. Even though such standards are easier to enforce for new buildings (Köppel and Ürge-Vorsatz, 2007), efforts should persist to ensure the implementation of the remediation duty for existing buildings. In addition, our simulations confirm that regulations don't encourage households to go beyond the standard's requirements; the threshold of these two regulatory instruments should thus be raised regularly. With the two latter instruments being included into the national policy mix, the further presence of capital grants and subsidised loans is essential. These financial instruments allow low-income homes to meet the standard's demands and incite households to go beyond the threshold. However, our results indicate that the design of these subsidy schemes is decisive for the tool's cost-effectiveness: the instrument's application period should be limited relative to the product's market dynamics and eligible households should be specified. To curb the rebound effect that is induced by these four instruments, taxes should not be omitted in the country's policy mix. Considering the overall effectiveness of evaluated tax instruments, we advise the government to focus on the implementation of a national carbon tax (and hereby set an important example for other EU Member States), and to maintain the energy tax rates at the required minimum level of the ETD. To reduce the adverse effects of such a taxation policy, that is, falling economic growth or competitiveness of heavy energy-using industries, the revenues of the tax should be used to promote energy conservation (Callan et al., 2009) (for example, by using the revenues to fund a part of the subsidy schemes).

Future research should focus on the set-up of an alternative modelling method where one considers the dwellings' thermal insulation class instead of their energy efficiency class, and where one adds information about the buildings' heating system into the model. That way, the determination of the building stock's final space heating energy demand could be improved, and dwellings that realise energy savings by solely replacing their heating system could be taken into account. Additionally, there is room for a better representation of households' behavioural patterns, for example, the decision-making behaviour and the adjustment factor could be improved by including additional socio-economic variables into the model. Such modifications should be realised once the needed data on Luxembourgish households are available. 


\section{A Appendix}

\section{A.1 The impacts of climate change on the heterogeneity parameter $\nu$}

Whenever the heterogeneity parameter $\nu$ increases, the price elasticity of demand (for us the elasticity of the number of retrofits) increases (in absolute value). Actually, we saw earlier (in equation (6)) that the number of retrofits from $i$ to $f$ is roughly proportional to the inverse of the life cycle costs $\operatorname{LCC}_{i, f, D, e_{i}}(\tau)$ of such a renovation raised to the power of $\nu$. This implies that if the life cycle costs $P$ of a retrofit increase by $100 \%$, the number $Q$ of retrofits decreases by $\left(2^{-\nu}-1\right) \times 100 \%$. The price elasticity at the initial price and initial number is hence

$$
\frac{\Delta Q / Q}{\Delta P / P}=\frac{\left(2^{-\nu}-1\right) \times 100 \%}{100 \%}
$$

Based on our assumption that the market becomes more heterogeneous over time (due to the impacts of climate change), we model $\nu$ as a decreasing logistic function of time with asymptotic values 7.5 and 1 :

$$
\nu(t)=1+\frac{6.5}{1+\exp (c t-d)} \quad(c>0) .
$$

Although climate summit meetings target zero emissions around 2050 and a limitation of global warming to less than 2 degrees Celsius by the end of this century (Falkner, 2016; Schleussner et al., 2016), recent developments resulted in forecasts that the effects of climate change will be seriously perceptible around 2030 (Pachauri et al., 2014). This is why we set the inflection point of the sigmoid curve at the year $2040(t=27)$. This means that $27 c=d+\ln 0.5$. This condition and the information $\nu(0)=7$ give $d=2.48$ and $c=0.066$ :

$$
\nu(t)=1+\frac{6.5}{1+\exp (0.066 t-2.48)} .
$$

In 2060 the value of $\nu$ is (a bit higher than) 3.25, which means that approximately $10 \%$ of the population maintain their renovation choice even when the costs double.

\section{A.2 Calibration of the initial intangible costs}

In order to calculate the initial intangible costs $\mathrm{IC}_{i, f}(0)$ we consider for any $i<B$ the system

$$
\mathrm{PR}_{i, f}(0)=F_{f}\left(\operatorname{INVC}_{i, h>i}(0), \operatorname{ENERC}_{h>i}(0), \operatorname{IC}_{i, h>i}(0)\right) \quad(i<f \leq A)
$$

which is obtained from equation (6) and (7).

We derive the proportions $\mathrm{PR}_{i, f}(0)$ from the analysis of 402 retrofitting operations undertaken in the Luxembourgish residential sector. This sample does not allow us to observe the proportions $\mathrm{PR}_{i, f} ; D, e_{i}(0)$. The initial energy costs must therefore also be independent of $D$ and $e_{i}$. The sample is split into two building types, individual houses and flats. In order to eliminate $D$ from the energy costs $\operatorname{ENERC}_{h, D, e_{i}}(0)$ we use a weighted mean $r$ of the average discount rates of the building types. To eliminate the carrier we calculate the proportions $\mathrm{PR}_{e_{i}}(0)$ from the available data and compute the energy costs in each efficiency class as weighted mean:

$$
\operatorname{ENERC}_{h}(0)=\sum_{e_{i}} \operatorname{PR}_{e_{i}}(0) P_{e_{i}}(0) \rho_{h, e_{i}} \sum_{\mathfrak{t}=1}^{N}(1+r)^{-\mathfrak{t}} .
$$


The sum of the proportions on the left hand side of (30) is equal to 1, just as the sum of the functions on the right hand side is (cf. equation (6)). For this reason, the system (30) reduces to the same system but with $f>i$ and $f<A$. The new system is a system of $8-i$ equations (cf. possible values of $f$ ) and of $9-i$ unknown intangible costs (cf. possible values of $h$ ). This is why an additional equation must be added to the new system. The percentage $\lambda$ of the average $\mathrm{LCC}_{i, h>i}(0)$ which consists of the average $\mathrm{IC}_{i, h>i}(0)$ is defined by equation

$$
\sum_{h>i} \mathrm{PR}_{i, h}(0) \mathrm{IC}_{i, h}(0)=\lambda \sum_{h>i} \mathrm{PR}_{i, h}(0) \mathrm{LCC}_{i, h}(0)
$$

in the same unknown intangible costs $\mathrm{IC}_{i, h>i}(0)$. This is the needed additional equation. It contains the parameter $\lambda$ which should of course have a low value. For any $i<B$ we use the fsolve command of MATLAB to find the lowest value of $\lambda$ for which the total system (new system and additional equation) has a solution. This finishes the calibration of the initial intangible costs.

\section{A.3 Decreasing the heterogeneity parameter $\nu$ in certain subpopulations}

We justified in the paragraph 3.3 that if $f \leq C$ only the carriers $\mathrm{F}, \mathrm{F}+\mathrm{s}, \mathrm{G}$ and $\mathrm{G}+\mathrm{s}$ are possible. If $f=B$ the decider can choose $e_{f} \in\{\mathrm{F}, \mathrm{F}+\mathrm{s}, \mathrm{G}, \mathrm{G}+\mathrm{s}, \mathrm{E}, \mathrm{E}+\mathrm{s}\}$, whereas for $f=A$ all eight carriers are possible final carriers. In the first case we choose in the calculation of the proportions PRS the parameter $\nu(\tau)$ given by equation (29). In the final class $A[B]$ we choose $\nu(\tau)-1$ $[\nu(\tau)-0.5]$ and further reduce this parameter in a way which depends on the chosen carrier.

In order to specify a coherent way to further reduce $\nu$ we record numerically, on a scale from 0 to 5 , the environmental awareness $\alpha$ of the deciders who switch in $A(B)$ to the carrier

$$
\begin{gathered}
e_{f}=\mathrm{F}(\mathrm{F}+\mathrm{s}, \mathrm{G}, \mathrm{G}+\mathrm{s}, \mathrm{E}, \mathrm{E}+\mathrm{s}, \mathrm{P} \text { or } \mathrm{P}+\mathrm{s}) \\
\left(e_{f}=\mathrm{F}(\mathrm{F}+\mathrm{s}, \mathrm{G}, \mathrm{G}+\mathrm{s}, \mathrm{E} \text { or } \mathrm{E}+\mathrm{s})\right) .
\end{gathered}
$$

We set

$$
\begin{gathered}
\alpha=0.0(0.4,0.4,0.8,3.2,3.6,4.0 \text { and } 4.4) \\
(\alpha=0.0(0.2,0.2,0.4,1.6 \text { and } 1.8)) .
\end{gathered}
$$

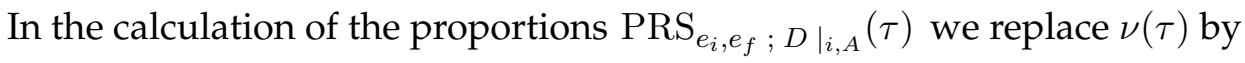

$$
\nu_{A}(\alpha, \tau)=\nu(\tau)-1-\pi \alpha
$$

The coefficient $\pi$ is determined by the request that for the maximal awareness 5 the heterogeneity parameter is $\nu_{A}(5,47)=1$ in the year 47 (that is, in 2060). We obtain

$$
\nu_{A}(\alpha, \tau)=\nu(\tau)-1-0.25 \alpha
$$

For $B$ we get

$$
\nu_{B}(\alpha, \tau)=\nu(\tau)-0.5-0.35 \alpha .
$$

Finally we use the formula (20) to find the proportions PRS in the classes $f \leq C$. In $f=A$ and $f=B$ we use the same formula but replace $\nu(\tau)$ by $\nu_{A}(\alpha, \tau)$ and $\nu_{B}(\alpha, \tau)$ and choose the 
value $\alpha$ that corresponds in $A$ and $B$ to the final carriers $e_{f}$ and $e_{h} \cdot{ }^{25}$

\section{A.4 Dynamic buidling types in new constructions}

The data of STATEC (2017) suggests that the percentage $\operatorname{PR}_{\mathrm{F}}(\tau)$ of flats in the new constructions is an increasing logistic function of the relative growth

$$
G(\tau)=\frac{H(\tau)-H(1960)}{H(1960)}
$$

of the total building stock with respect to 1960:

$$
\operatorname{PR}_{\mathrm{F}}(\tau)=a+\frac{b}{1+\exp (-c G(\tau)+d)} \quad(a, b, c, d>0) .
$$

We have

$$
\lim _{G \rightarrow+\infty} \mathrm{PR}_{\mathrm{F}}=a+b=1
$$

and set

$$
\lim _{G \rightarrow-\infty} \mathrm{PR}_{\mathrm{F}}=a=0 .
$$

This choice is justified as the linear regression that gives $c(c=0.54)$ and $d(d=0.42)$ is of good quality and the law

$$
\operatorname{PR}_{\mathrm{F}}(\tau)=\frac{1}{1+\exp (-0.54 G(\tau)+0.42)}
$$

leads to a good approximation of the observed value $\operatorname{PR}_{\mathrm{F}}(1960)$.

\footnotetext{
${ }^{25}$ This alternative modelling approach produces good (in particular not at all excessive) results.
} 


\section{A.5 Figures and Tables}

Table 5 - Final energy consumption and direct $\mathrm{CO}_{2}$ emissions (total building stock)

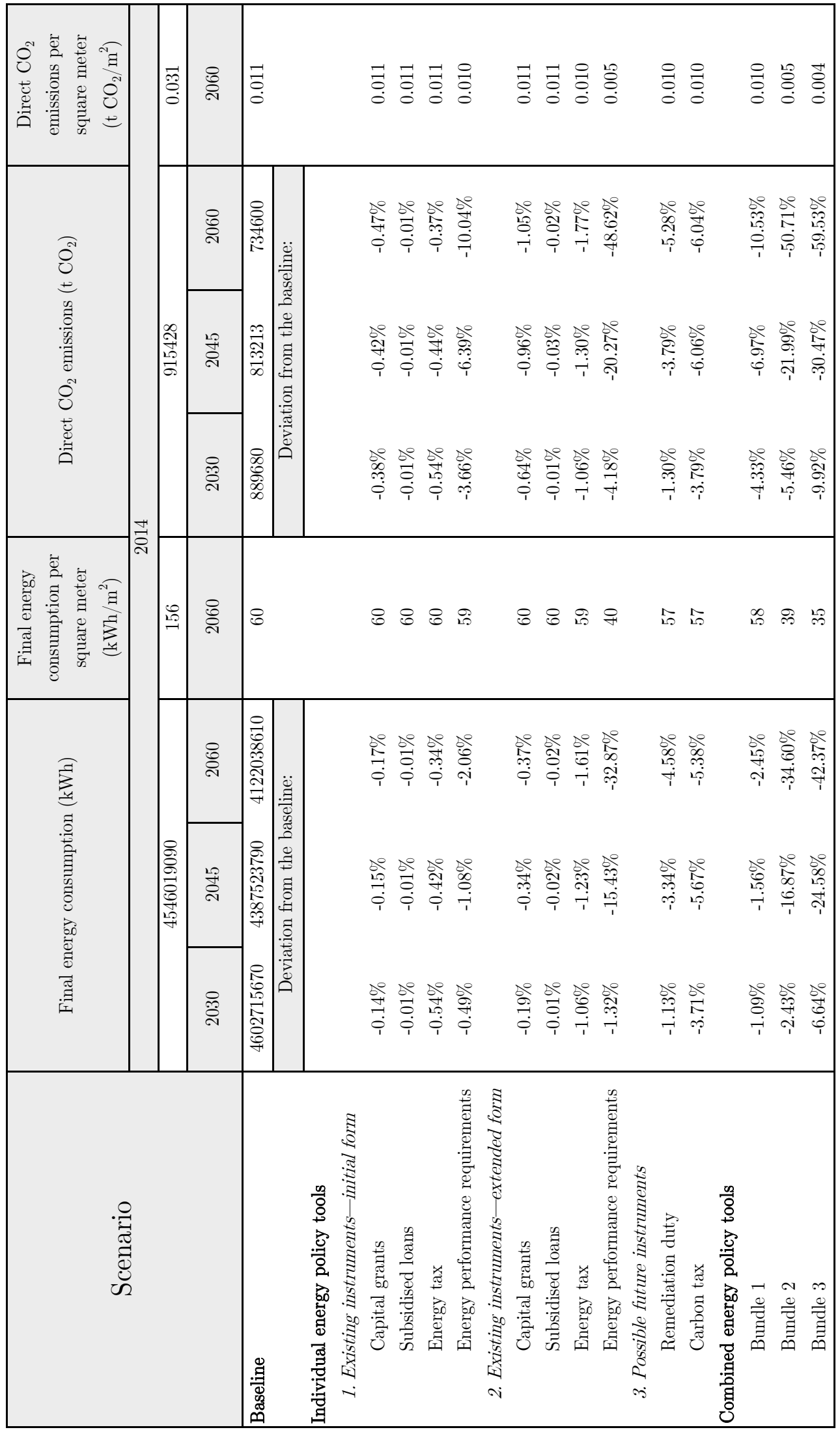


Table 6 - Number of retrofits, Conventional energy consumption, and Adjustment factor (total building stock)

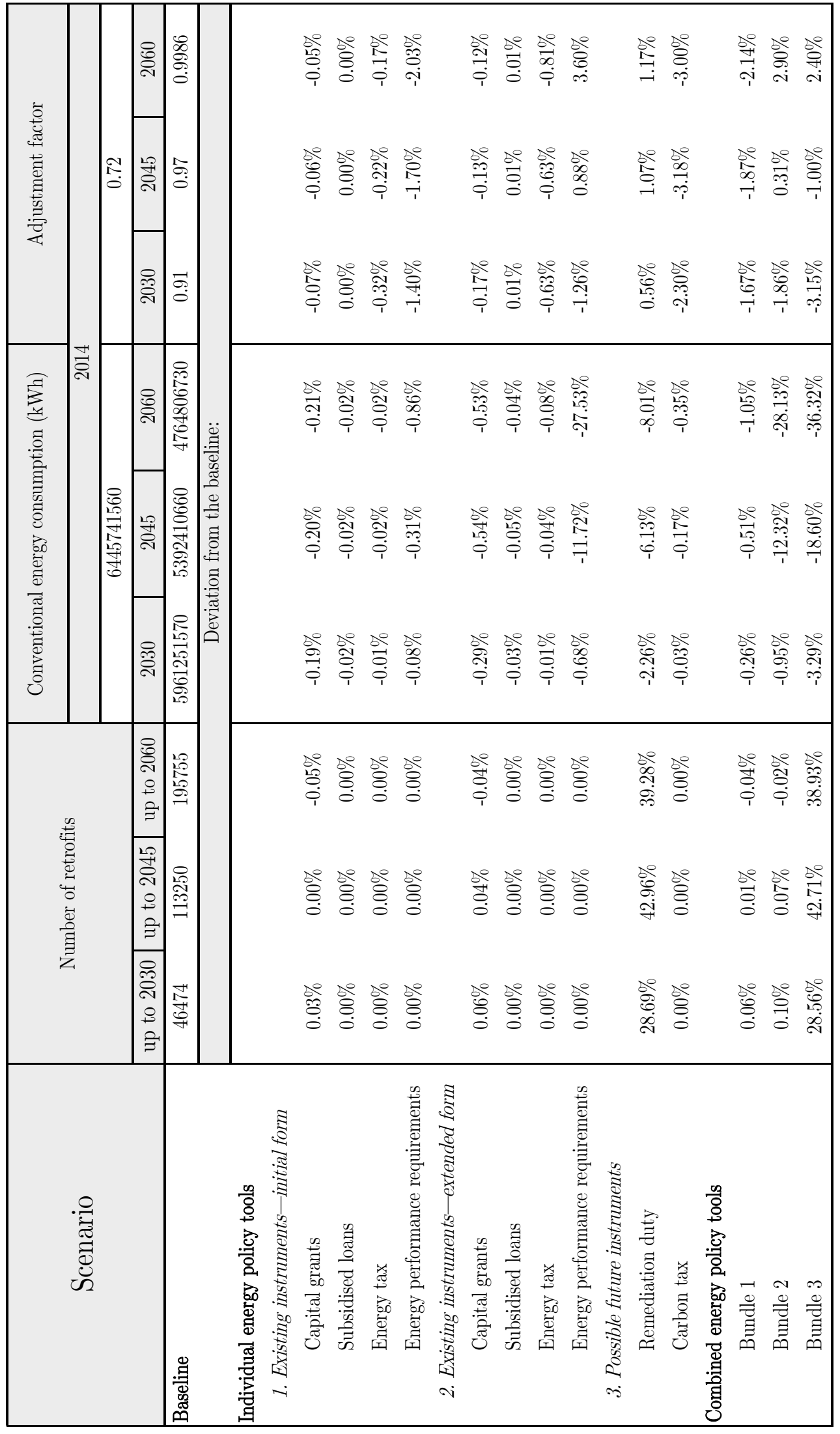


Table 7 - Share of dwellings per energy class and carrier (total building stock of 2060)

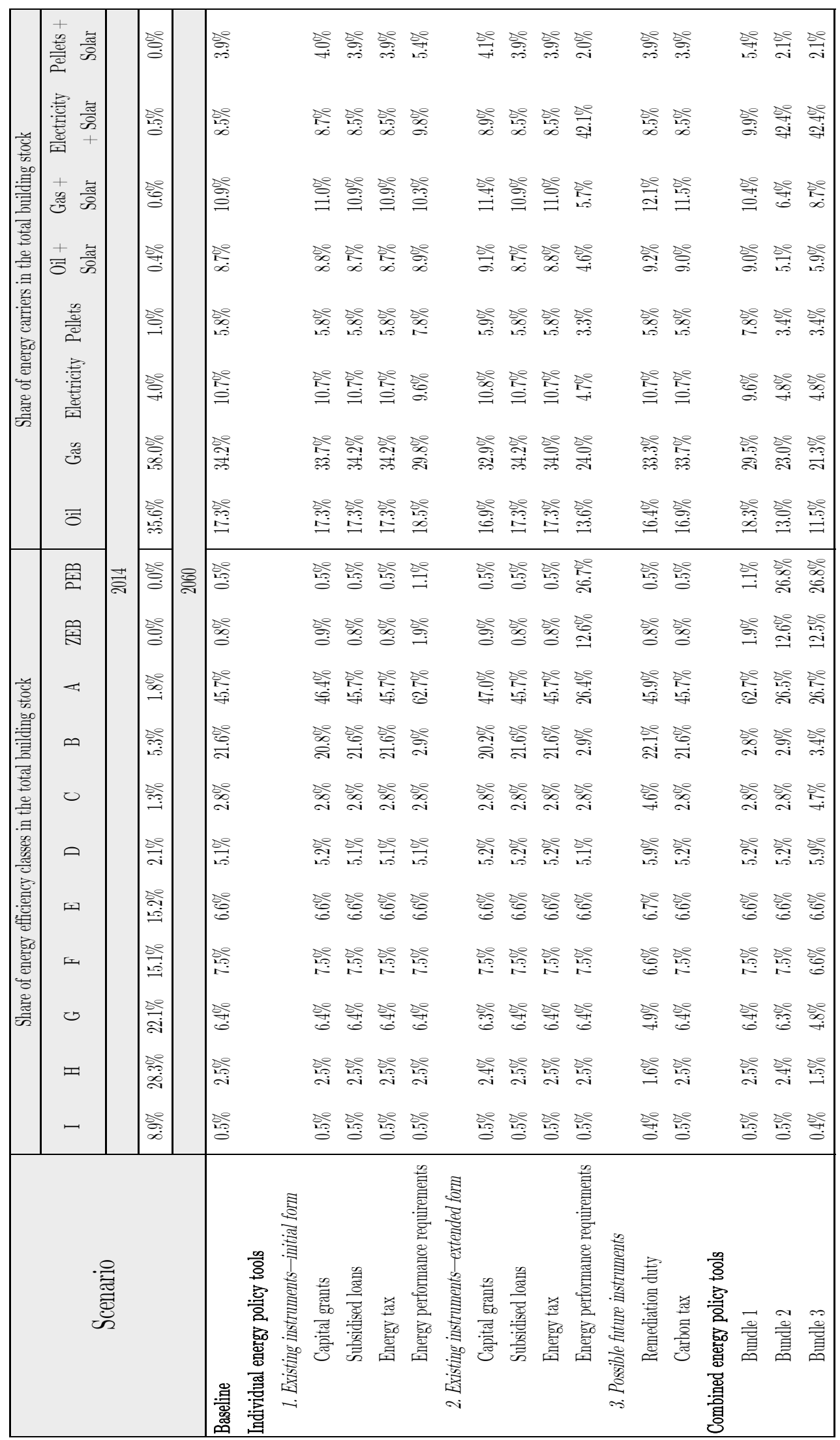


Table 8 - Benefit to cost ratio (in $€$; from a governmental perspective)

\begin{tabular}{|c|c|c|c|c|}
\hline \multirow{2}{*}{ Scenario } & Direct revenues & Direct expenses & \multirow[t]{2}{*}{ Balance } & \multirow{2}{*}{$\begin{array}{l}\text { Benefit to cost ratio } \\
\qquad(€ / \mathrm{kWh} \text { saved })\end{array}$} \\
\hline & \multicolumn{2}{|c|}{ during instrument's time period } & & \\
\hline Baseline & 0.00 & 0.00 & 0.00 & 0.00 \\
\hline \multicolumn{5}{|l|}{ Individual energy policy tools } \\
\hline \multicolumn{5}{|l|}{ 1. Existing instruments - initial form } \\
\hline Capital grants & 0.00 & 506140246.29 & -506140246.29 & -74.10 \\
\hline Subsidised loans & 0.00 & 38398742.03 & -38398742.03 & -100.51 \\
\hline Energy tax & 199761810.82 & 0.00 & 199761810.82 & 14.33 \\
\hline Energy performance requirements & 0.00 & 0.00 & 0.00 & 0.00 \\
\hline \multicolumn{5}{|l|}{ 2. Existing instruments - extended form } \\
\hline Capital grants & 0.00 & 1871412395.10 & -1871412395.10 & -123.79 \\
\hline Subsidised loans & 0.00 & 123503822.62 & -123503822.62 & -135.61 \\
\hline Energy tax & 531075380.17 & 0.00 & 531075380.17 & 8.02 \\
\hline Energy performance requirements & 0.00 & 0.00 & 0.00 & 0.00 \\
\hline \multicolumn{5}{|l|}{ 3. Possible future instruments } \\
\hline Remediation duty & 0.00 & 0.00 & 0.00 & 0.00 \\
\hline Carbon tax & 2251340323.00 & 0.00 & 2251340323.00 & 10.15 \\
\hline \multicolumn{5}{|l|}{ Combined energy policy tools } \\
\hline Bundle 1 & 189813712.28 & 734329463.99 & -544515751.71 & -5.40 \\
\hline Bundle 2 & 451734598.71 & 2745141617.20 & -2293407018.49 & -1.61 \\
\hline Bundle 3 & 1776900472.89 & 2926571465.93 & -1149670993.04 & -0.66 \\
\hline
\end{tabular}

Table 9 - Energy consumption (comp. to 2007), $\mathrm{CO}_{2}$ emissions (comp. to 2005)

\begin{tabular}{|c|c|c|c|c|c|c|}
\hline \multirow{2}{*}{ Scenario } & \multicolumn{3}{|c|}{ Final energy consumption compared to 2007} & \multicolumn{3}{|c|}{ Direct $\mathrm{CO}_{2}$ emissions compared to 2005} \\
\hline & \begin{tabular}{|c|}
2020 \\
Target : $-20 \%$ \\
\end{tabular} & 2030 & 2060 & $\begin{array}{c}2020 \\
\text { Target: }-20 \%\end{array}$ & $\begin{array}{c}2030 \\
\text { Target: }-40 \%\end{array}$ & 2060 \\
\hline Baseline & $28.79 \%$ & $18.97 \%$ & $6.55 \%$ & $6.76 \%$ & $-4.44 \%$ & $-21.10 \%$ \\
\hline \multirow{2}{*}{\multicolumn{7}{|c|}{$\begin{array}{l}\text { Individual energy policy tools } \\
\text { 1. Existing instruments - initial form }\end{array}$}} \\
\hline & & & & & & \\
\hline Capital grants & $28.68 \%$ & $18.81 \%$ & $6.37 \%$ & $6.46 \%$ & $-4.80 \%$ & $-21.47 \%$ \\
\hline Subsidised loans & $28.79 \%$ & $18.96 \%$ & $6.54 \%$ & $6.76 \%$ & $-4.45 \%$ & $-21.11 \%$ \\
\hline Energy tax & $27.80 \%$ & $18.33 \%$ & $6.19 \%$ & $5.96 \%$ & $-4.96 \%$ & $-21.39 \%$ \\
\hline Energy performance requirements & $28.37 \%$ & $18.39 \%$ & $4.35 \%$ & $4.63 \%$ & $-7.94 \%$ & $-29.02 \%$ \\
\hline \multicolumn{7}{|l|}{ 2. Existing instruments - extended form } \\
\hline Capital grants & $28.66 \%$ & $18.75 \%$ & $6.16 \%$ & $6.44 \%$ & $-5.05 \%$ & $-21.93 \%$ \\
\hline Subsidised loans & $28.79 \%$ & $18.96 \%$ & $6.53 \%$ & $6.76 \%$ & $-4.45 \%$ & $-21.12 \%$ \\
\hline Energy tax & $27.80 \%$ & $17.71 \%$ & $4.84 \%$ & $5.96 \%$ & $-5.46 \%$ & $-22.49 \%$ \\
\hline Energy performance requirements & $28.37 \%$ & $17.41 \%$ & $-28.47 \%$ & $4.63 \%$ & $-8.44 \%$ & $-59.46 \%$ \\
\hline \multicolumn{7}{|l|}{ 3. Possible future instruments } \\
\hline Remediation duty & $28.79 \%$ & $17.63 \%$ & $1.67 \%$ & $6.76 \%$ & $-5.68 \%$ & $-25.26 \%$ \\
\hline Carbon tax & $28.79 \%$ & $14.56 \%$ & $0.82 \%$ & $6.76 \%$ & $-8.06 \%$ & $-25.86 \%$ \\
\hline \multicolumn{7}{|l|}{ Combined energy policy tools } \\
\hline Bundle 1 & $27.37 \%$ & $17.68 \%$ & $3.94 \%$ & $3.75 \%$ & $-8.58 \%$ & $-29.41 \%$ \\
\hline Bundle 2 & $27.35 \%$ & $16.09 \%$ & $-30.32 \%$ & $3.73 \%$ & $-9.66 \%$ & $-61.11 \%$ \\
\hline Bundle 3 & $27.35 \%$ & $11.07 \%$ & $-38.60 \%$ & $3.73 \%$ & $-13.92 \%$ & $-68.07 \%$ \\
\hline
\end{tabular}


Figure 1 - Evolution of the existing and new building stock

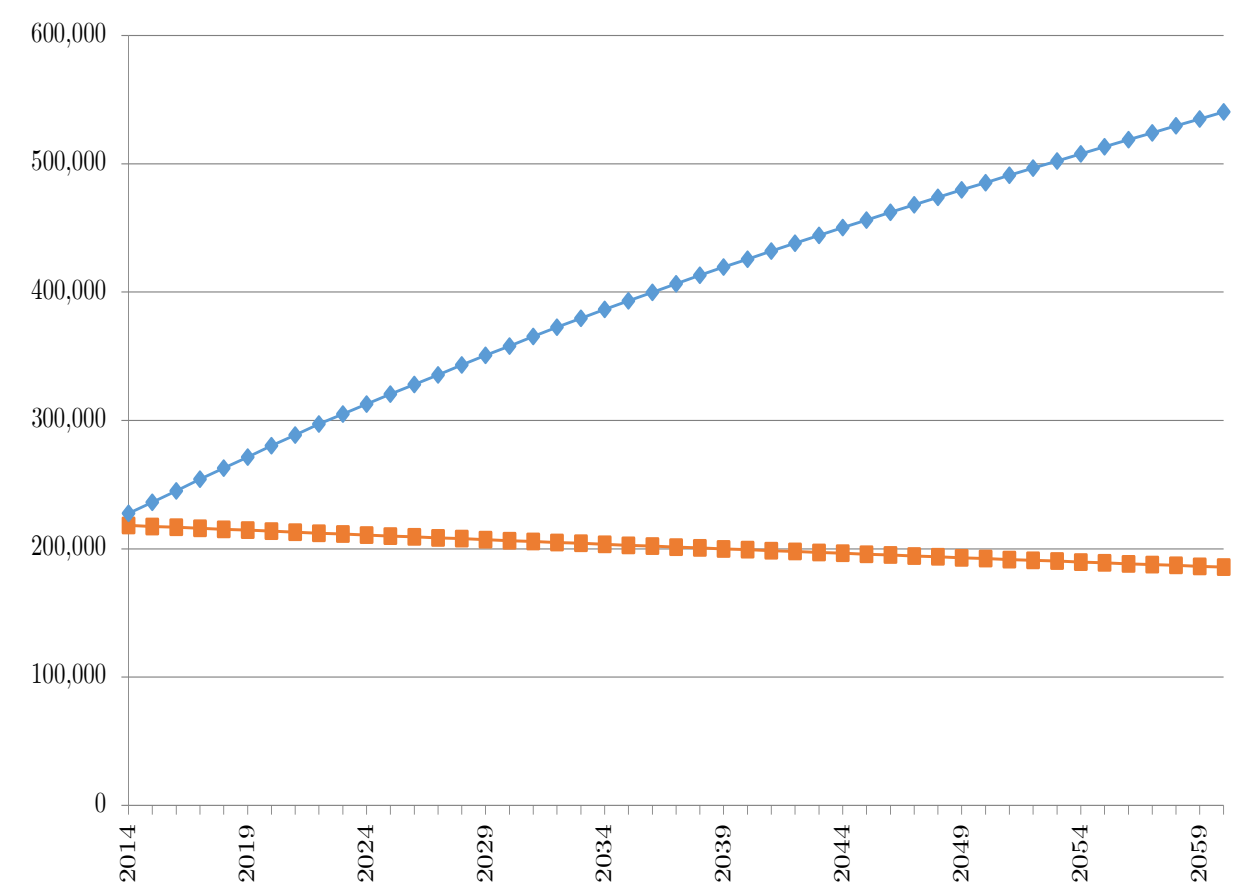

$\bullet$ New building stock

- Existing building stock

Figure 2 - Adjustment factor (total building stock)

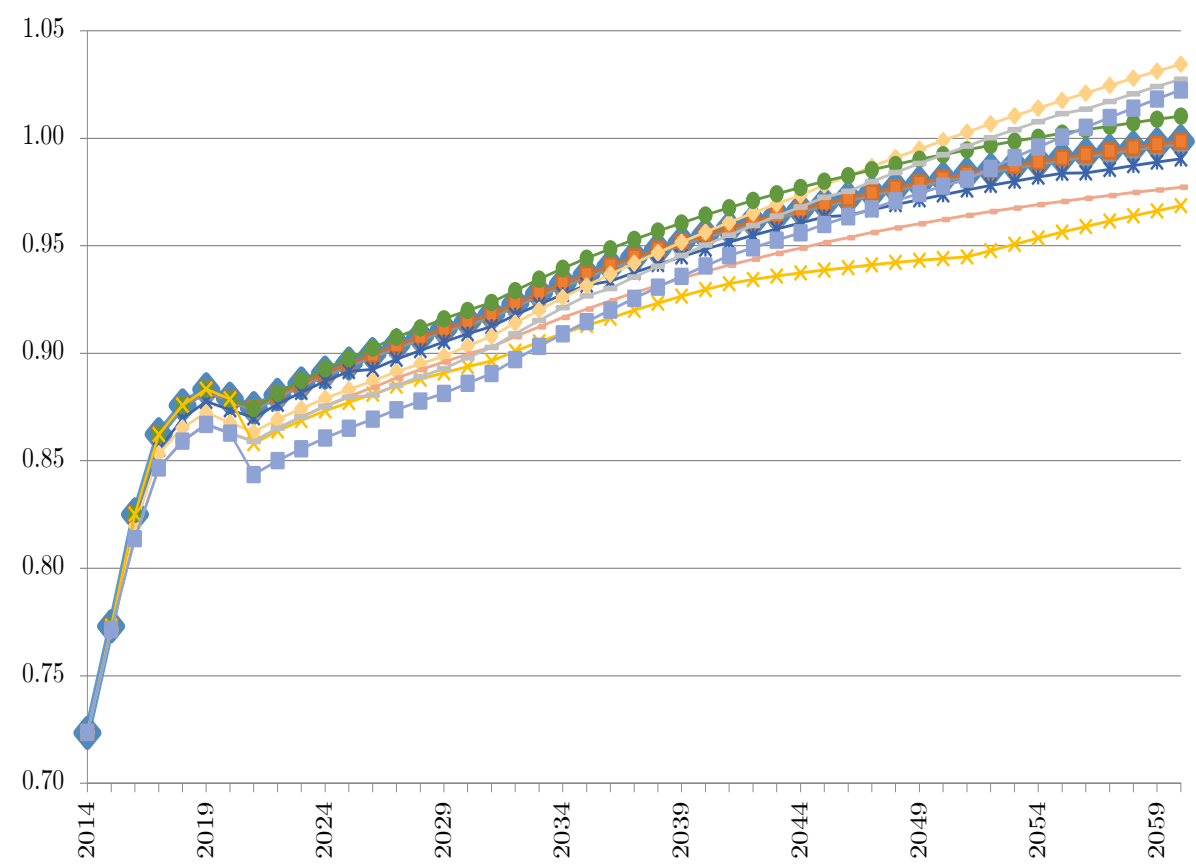

$\sim$ Baseline

$\neg$ Capital grants

$\neg$ Subsidised loans

*-Energy tax

$\_$Energy performance requirements

- Remediation duty

* Carbon tax

$=$ Bundle 1

- Bundle 2

- - Bundle 3 
Figure 3 - Final energy consumption (kWh) (total building stock)

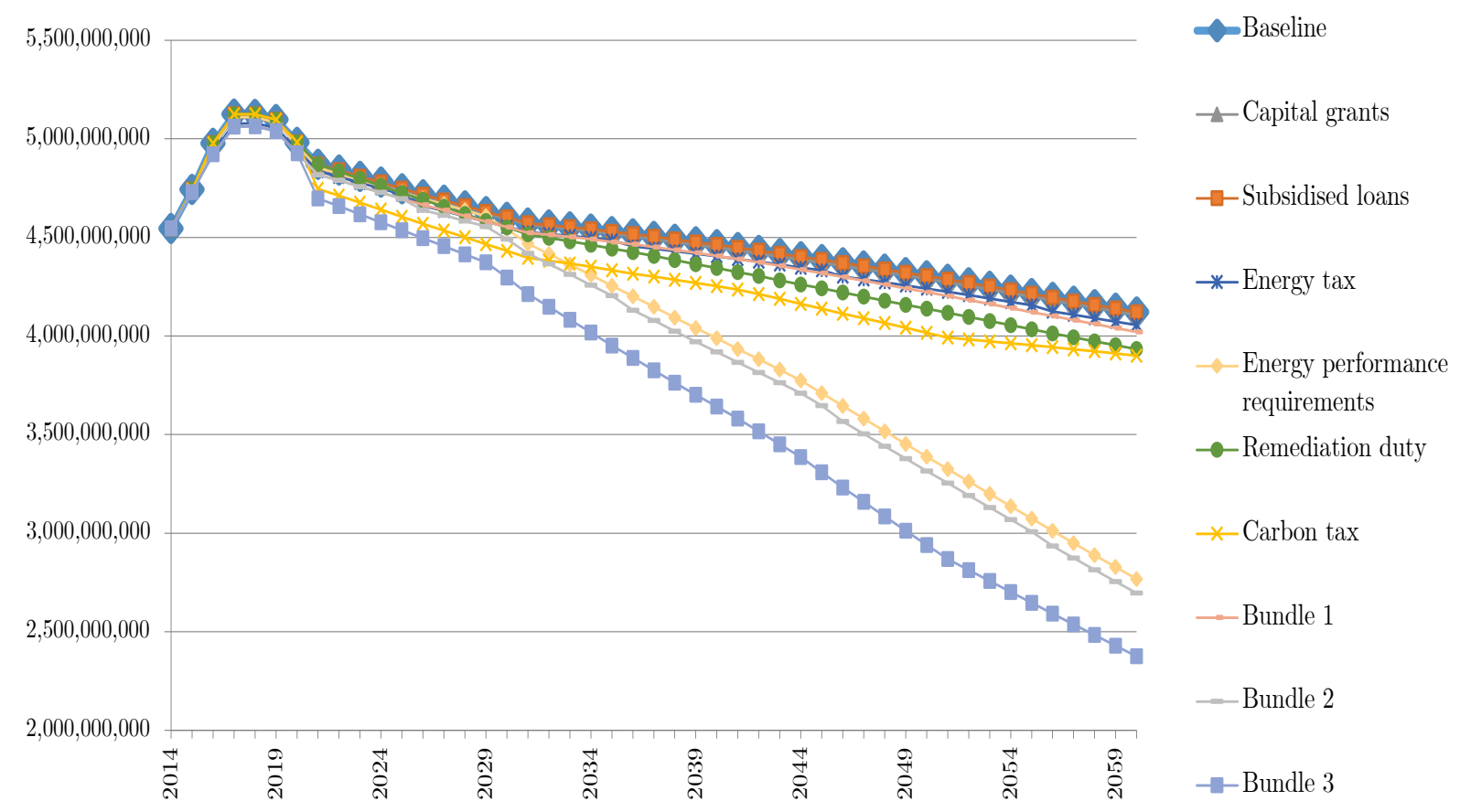

Figure 4 - Direct $\mathrm{CO}_{2}$ emissions ( $\left.\mathrm{t}_{2}\right)$ (total building stock)

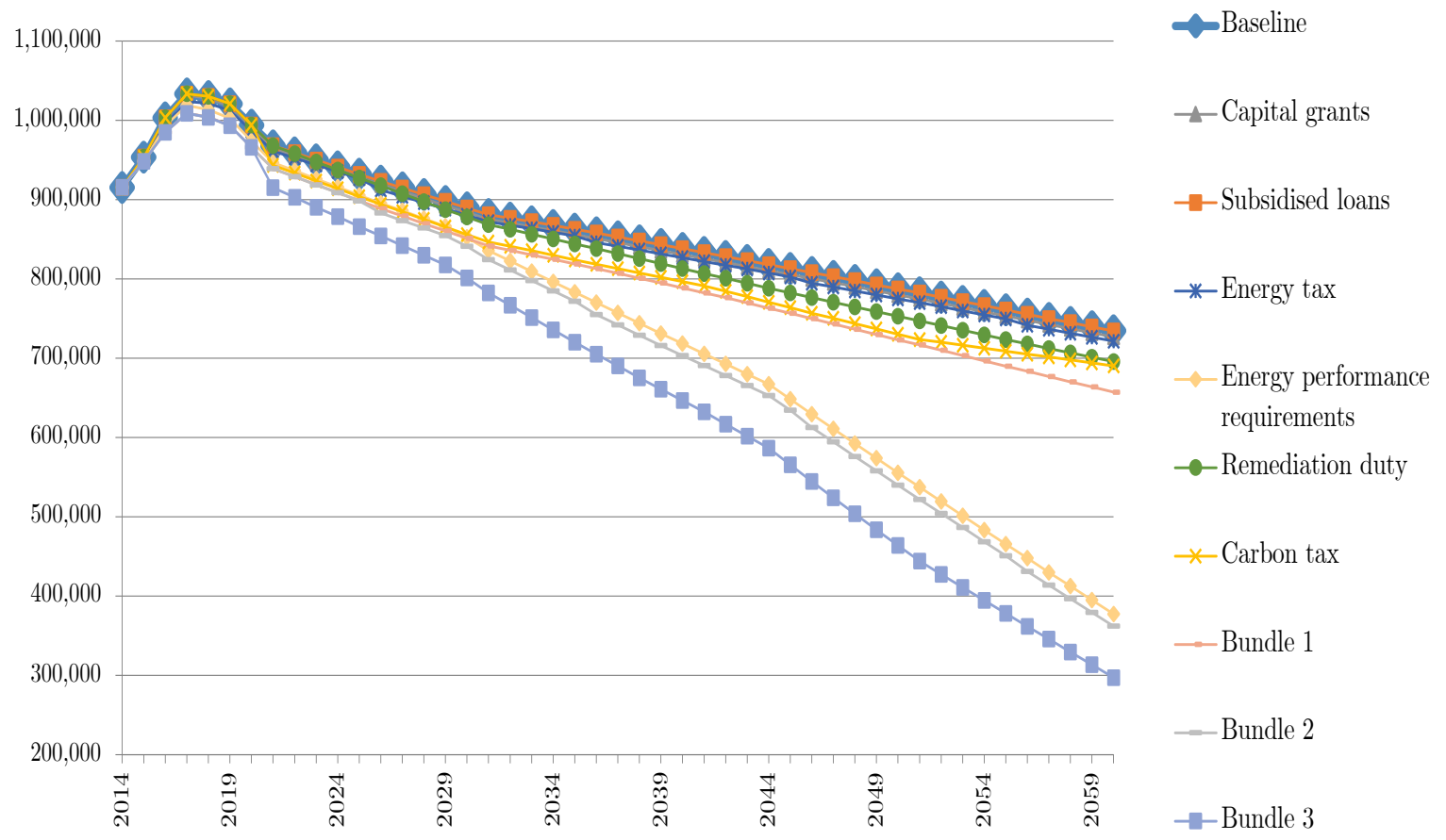


Figure 5 - Number of dwellings per energy class (total building stock of 2060)

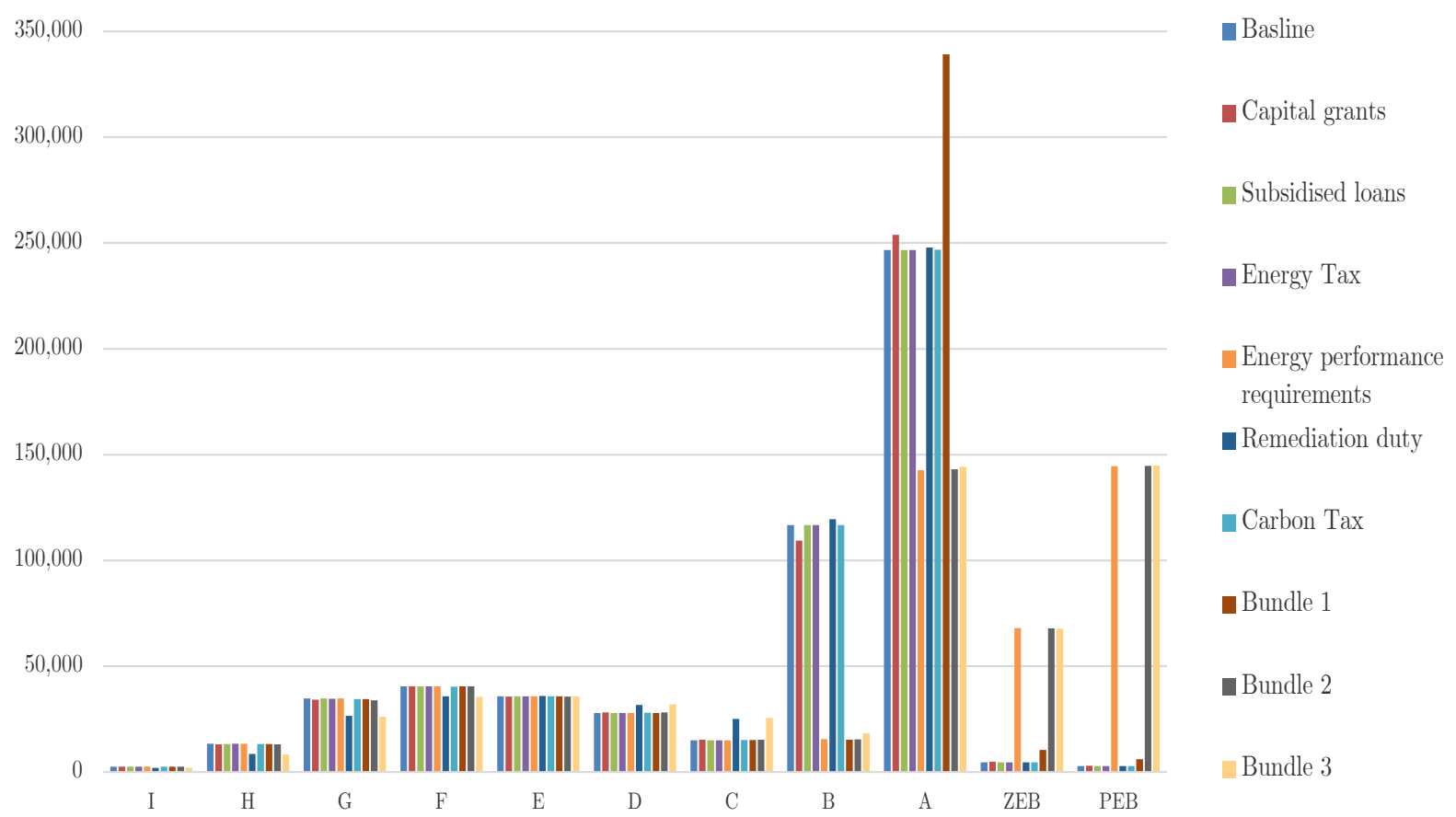

Figure 6 - Number of dwellings per energy carrier (total building stock of 2060)

Basline

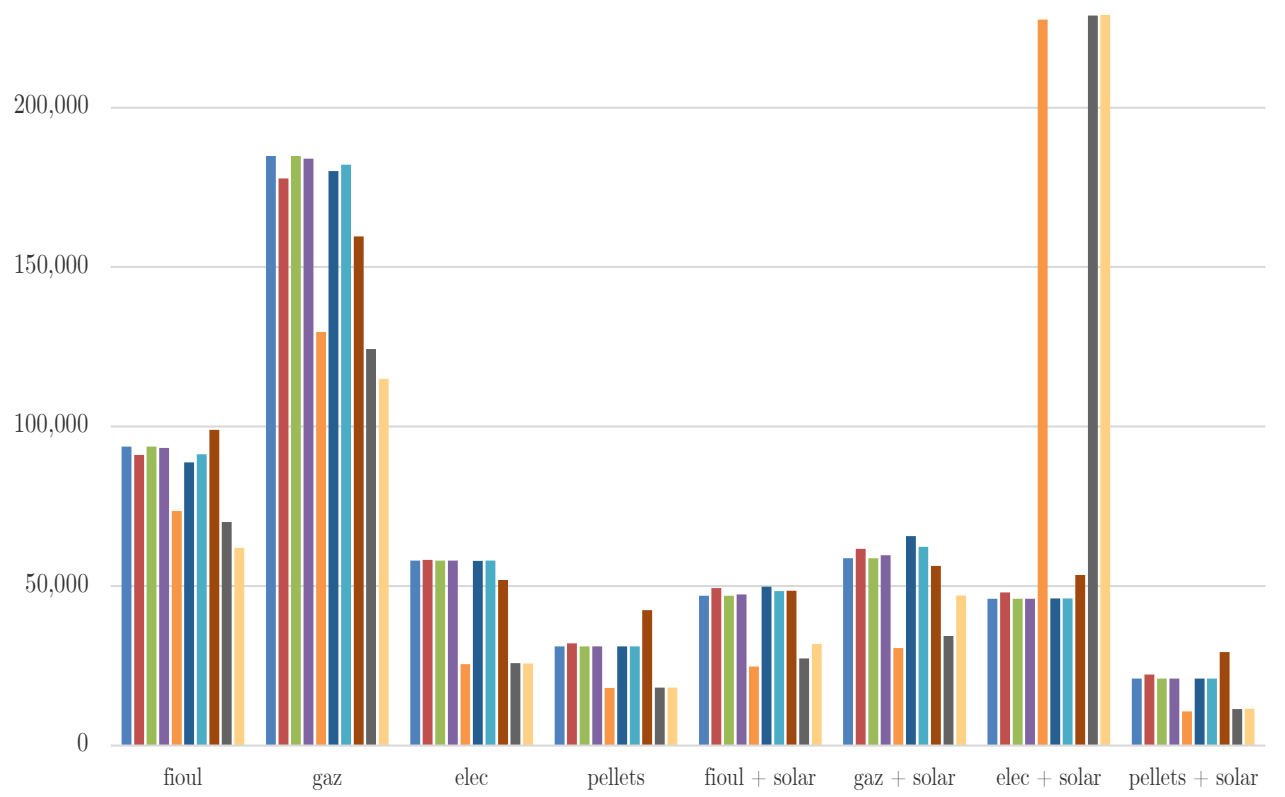

- Capital grants

- Subsidised loans

Energy Tax

Energy performance requirements

- Remediation duty

- Carbon Tax

Bundle 1

Bundle 2

Bundle 3 


\section{Figure 7 - Number of retrofitted dwellings}

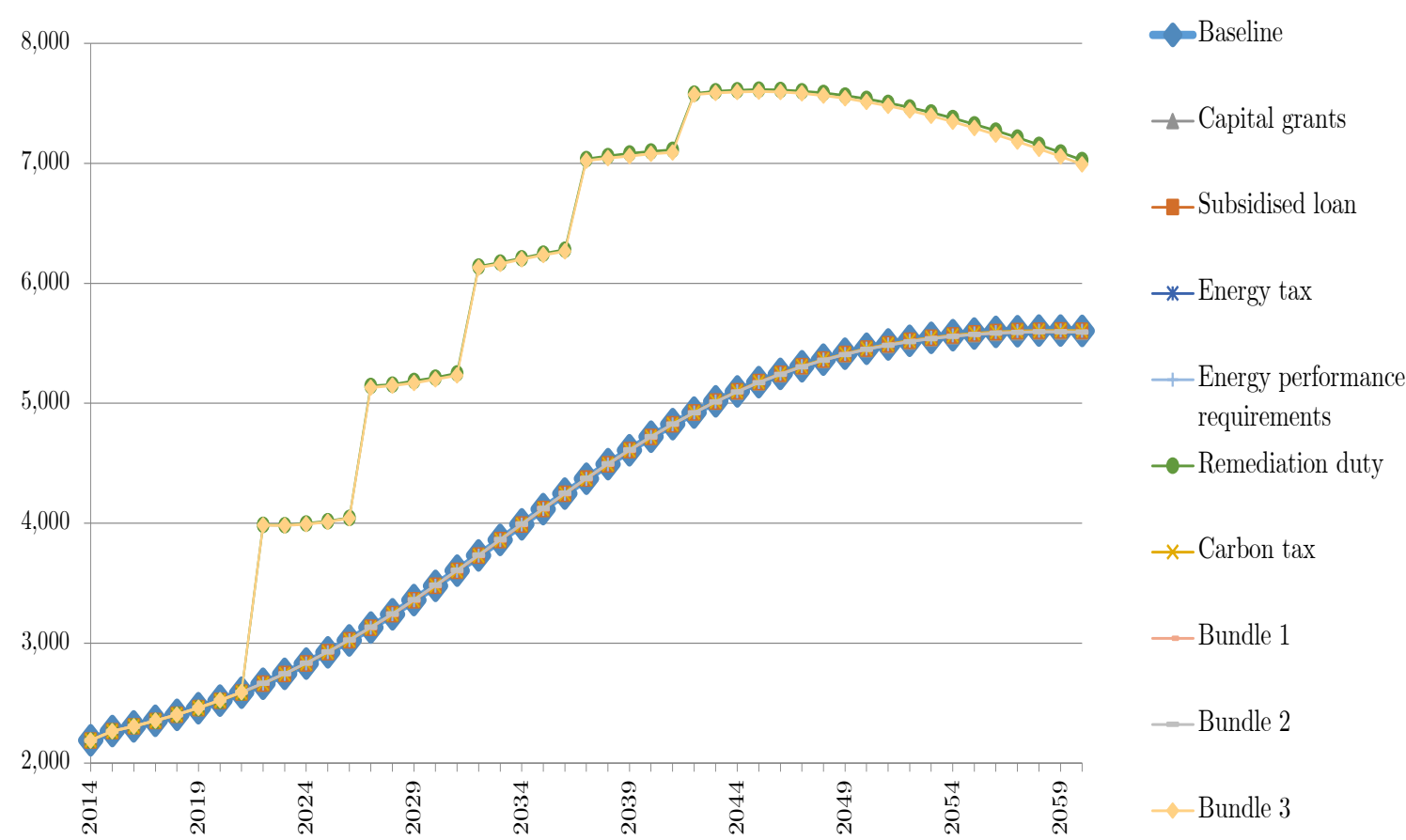

Figure 8 - Final energy consumption (kWh) (new building stock)

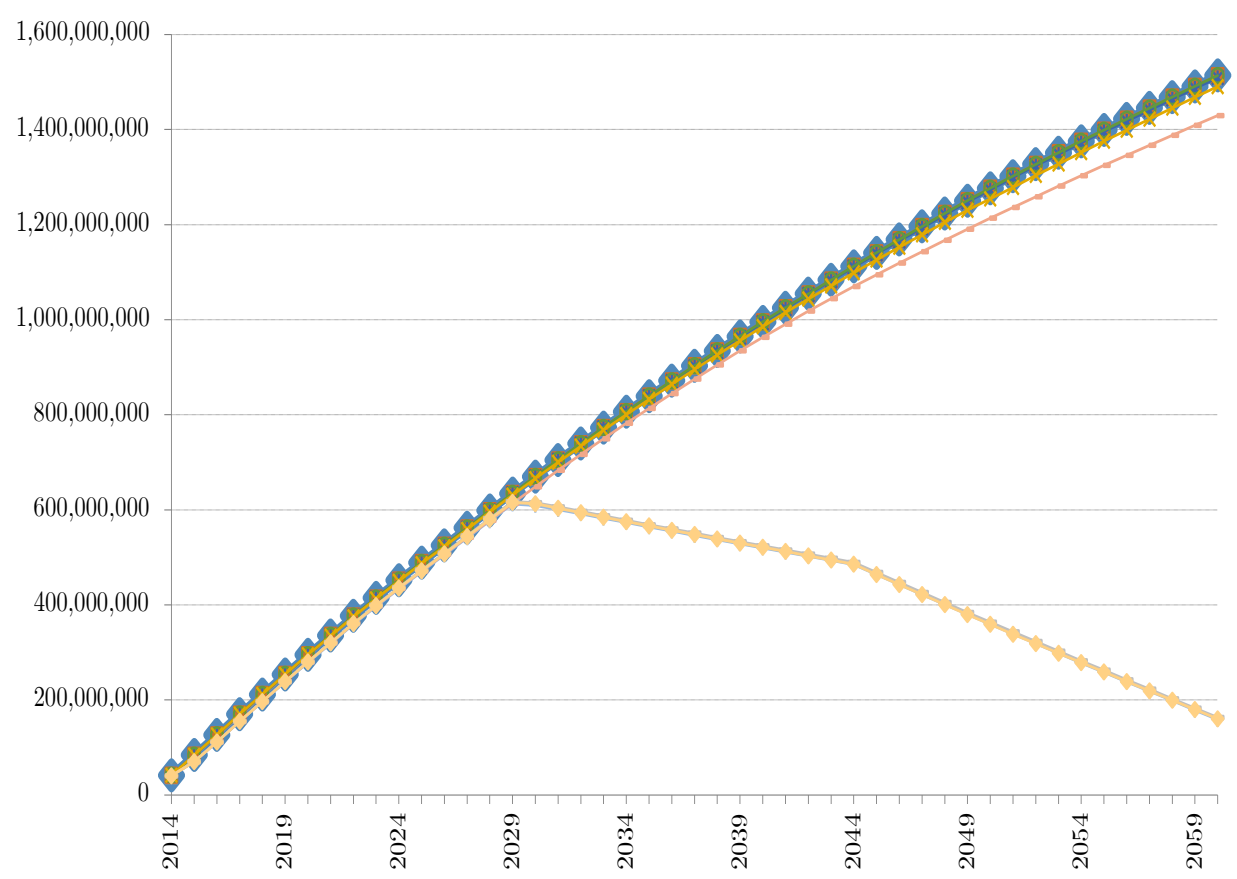

$\sim$ Baseline

$\rightarrow$ Capital grants

- - Subsidised loans

*-Energy Tax

+ Energy performance requirements

○-Remediation duty

$*$ Carbon Tax

$=$ Bundle 1

- Bundle 2

$\neg$ Bundle 3 


\section{References}

ADEME (2013). Les chiffres clés du bâtiment, pages 1-91.

Aminrad, Z., Zakaria, S., and Hadi, A. S. (2011). Influence of age and level of education on environmental awareness and attitude: case study on Iranian students in Malaysian Universities. The Social Sciences, 6(1):15-19.

Amstalden, R. W., Kost, M., Nathani, C., and Imboden, D. M. (2007). Economic potential of energy-efficient retrofitting in the Swiss residential building sector: The effects of policy instruments and energy price expectations. Energy Policy, 35(3):1819-1829.

Ástmarsson, B., Jensen, P. A., and Maslesa, E. (2013). Sustainable renovation of residential buildings and the landlord/tenant dilemma. Energy Policy, 63:355-362.

BBSR (2016). Mietrecht und energetische Sanierung im europäischen Vergleich. BBSR Online Publikation, (13):1-146.

Birol, F. et al. (2010). World Energy Outlook 2010. International Energy Agency, 1(3):1-738.

Boeters, S. and Koornneef, J. (2011). Supply of renewable energy sources and the cost of EU climate policy. Energy Economics, 33(5):1024-1034.

Böhringer, C., Koschel, H., and Moslener, U. (2008). Efficiency losses from overlapping regulation of EU carbon emissions. Journal of Regulatory Economics, 33(3):299-317.

Boonekamp, P. G. (2006). Actual interaction effects between policy measures for energy efficiency-a qualitative matrix method and quantitative simulation results for households. Energy, 31(14):2848-2873.

Bovenberg, A. L., Goulder, L. H., and Gurney, D. J. (2005). Efficiency costs of meeting industrydistributional constraints under environmental permits and taxes. RAND Journal of Economics, 36(4):951-971.

Bruvoll, A. and Larsen, B. M. (2004). Greenhouse gas emissions in Norway: Do carbon taxes work? Energy Policy, 32(4):493-505.

Bye, B., Fæhn, T., and Rosnes, O. (2018). Residential energy efficiency policies: Costs, emissions and rebound effects. Energy, 143:191-201.

Callan, T., Lyons, S., Scott, S., Tol, R. S., and Verde, S. (2009). The distributional implications of a carbon tax in Ireland. Energy Policy, 37(2):407-412.

Capros, P., De Vita, A., Tasios, N., Siskos, P., Kannavou, M., Petropoulos, A., Evangelopoulou, S., Zampara, M., Papadopoulos, D., Nakos, C., et al. (2016). EU Reference Scenario 2016: Energy, transport and GHG emissions-Trends to 2050. pages 1-221.

Cayre, E., Allibe, B., Laurent, M.-H., and Osso, D. (2011). There are people in the house! How the results of purely technical analysis of residential energy consumption are misleading for energy policies. Proceedings of the ECEEE Summer Study, Paper 7-277:1675-1683.

Chakravorty, U., Roumasset, J., and Tse, K. (1997). Endogenous substitution among energy resources and global warming. Journal of Political Economy, 105(6):1201-1234. 
Charlier, D. (2015). Energy efficiency investments in the context of split incentives among French households. Energy Policy, 87:465-479.

European Commission (2010). Energy 2020: A strategy for competitive, sustainable and secure energy. Communication from the Commission to the European Parliament and others, pages 1-20.

Eurostat (2017). European Union Statistics on Income and Living Conditions (EU-SILC).

Falkner, R. (2016). The Paris Agreement and the new logic of international climate politics. International Affairs, 92(5):1107-1125.

Fankhauser, S., Hepburn, C., and Park, J. (2010). Combining multiple climate policy instruments: how not to do it. Climate Change Economics, 1(03):209-225.

Fleiter, T., Worrell, E., and Eichhammer, W. (2011). Barriers to energy efficiency in industrial bottom-up energy demand models-A review. Renewable and sustainable energy reviews, 15(6):3099-3111.

Flues, F., Löschel, A., Lutz, B. J., and Schenker, O. (2014). Designing an EU energy and climate policy portfolio for 2030: Implications of overlapping regulation under different levels of electricity demand. Energy Policy, 75:91-99.

Geller, H., Harrington, P., Rosenfeld, A. H., Tanishima, S., and Unander, F. (2006). Polices for increasing energy efficiency: Thirty years of experience in OECD countries. Energy policy, 34(5):556-573.

Gerlagh, R. and Van der Zwaan, B. (2006). Options and Instruments for a Deep Cut in $\mathrm{CO}_{2}$ Emissions: Carbon Dioxide Capture or Renewables, Taxes or Subsidies? The Energy Journal, 27(3):25-48.

Ghalwash, T. (2007). Energy taxes as a signaling device: An empirical analysis of consumer preferences. Energy Policy, 35(1):29-38.

Gillingham, K., Harding, M., and Rapson, D. (2012). Split incentives in residential energy consumption. The Energy Journal, 33(2):37-62.

Gillingham, K., Keyes, A., and Palmer, K. (2018). Advances in evaluating energy efficiency policies and programs. Annual Review of Resource Economics, 10:511-532.

Gillingham, K. and Palmer, K. (2014). Bridging the energy efficiency gap: Policy insights from economic theory and empirical evidence. Review of Environmental Economics and Policy, $8(1): 18-38$.

Giraudet, L.-G., Branger, F., Guivarch, C., and Quirion, P. (2015). Global sensitivity analysis of an energy-economy model of the residential building sector. Environmental Modelling $\mathcal{E}$ Software, 70:45-54.

Giraudet, L.-G., Guivarch, C., and Quirion, P. (2011). Comparing and combining energy saving policies: Will proposed residential sector policies meet French official targets? The Energy Journal, 32:213-242.

Giraudet, L.-G., Guivarch, C., and Quirion, P. (2012). Exploring the potential for energy conservation in French households through hybrid modeling. Energy Economics, 34(2):426445. 
Haas, T. and Peltier, F. (2017). Projections macroéconomiques et démographiques de long terme: 2017-2060. Bulletin du STATEC, (3):1-53.

Hausman, J. A. (1979). Individual discount rates and the purchase and utilization of energyusing durables. Bell journal of Economics, 10(1):33-54.

Högberg, L. (2013). The impact of energy performance on single-family home selling prices in Sweden. Journal of European Real Estate Research, 6(3):242-261.

Hourcade, J.-C., Jaccard, M., Bataille, C., and Ghersi, F. (2006). Hybrid modeling: New answers to old challenges. Introduction to the special issue of 'The Energy Journal'. The Energy Journal, 27:1-11.

Huang, P., Zhang, X., and Deng, X. (2006). Survey and analysis of public environmental awareness and performance in Ningbo, China: A case study on household electrical and electronic equipment. Journal of Cleaner Production, 14(18):1635-1643.

Itard, L. (2008). Towards a sustainable Northern European housing stock: Figures, facts, and future. IOS Press, pages 1-213.

Jaccard, M. and Dennis, M. (2006). Estimating home energy decision parameters for a hybrid energy-economy policy model. Environmental Modeling $\mathcal{E}$ Assessment, 11(2):91-100.

Jaffe, A. B., Newell, R. G., and Stavins, R. N. (2004). Economics of energy efficiency. Encyclopedia of Energy, 2:79-90.

Jaffe, A. B. and Stavins, R. N. (1994). The energy-efficiency gap. What does it mean? Energy Policy, 22(10):804-810.

Knobloch, F., Pollitt, H., Chewpreecha, U., and Mercure, J.-F. (2019). Simulating the deep decarbonisation of residential heating for limiting global warming to $1.5 \mathrm{C}^{\circ}$. Energy Efficiency, 12(2):521-550.

Köppel, S. and Ürge-Vorsatz, D. (2007). Assessment of policy instruments for reducing greenhouse gas emissions from buildings. Report for the UNEP-Sustainable Buildings and Construction Initiative. United Nations Environment Programme and Central European University, pages 1-91.

Lechtenböhmer, S. and Schüring, A. (2011). The potential for large-scale savings from insulating residential buildings in the EU. Energy Efficiency, 4(2):257-270.

Lee, W. and Yik, F. (2004). Regulatory and voluntary approaches for enhancing building energy efficiency. Progress in Energy and Combustion Science, 30(5):477-499.

Levine, M., Ürge-Vorsatz, D., Blok, K., Geng, L., Harvey, D., Lang, S., Levermore, G., Mongameli Mehlwana, A., Mirasgedis, S., Novikova, A., et al. (2007). Residential and commercial buildings. Climate Change, 20:1-17.

Lin, B. and Li, X. (2011). The effect of carbon tax on per capita $\mathrm{CO}_{2}$ emissions. Energy Policy, 39(9):5137-5146. 
Lindner, M., Maroschek, M., Netherer, S., Kremer, A., Barbati, A., Garcia-Gonzalo, J., Seidl, R., Delzon, S., Corona, P., Kolström, M., et al. (2010). Climate change impacts, adaptive capacity, and vulnerability of European forest ecosystems. Forest Ecology and Management, 259(4):698-709.

Matteoli, A. (2003). Council Directive 2003/96/EC of 27 October 2003 restructuring the Community framework for the taxation of energy products and electricity. Official Journal of the European Union, 33(1):41-70.

McCormick, K. and Neij, L. (2009). Experience of policy instruments for energy efficiency in buildings in the Nordic countries. Lund University, pages 1-74.

Ministry of the Economy of Luxembourg (2017). Energiepass-Datenbank des Wirtschaftsministeriums Luxemburg.

Ministry of the Economy of Luxembourg and Lichtmeß, M. (2014). Berechnung kostenoptimaler Niveaus von Mindestanforderungen an die Gesamtenergieeffizienz für neue und bestehende Wohn- und Nichtwohngebäude. Governmental Publication, pages 1-128.

Ministry of the Economy of Luxembourg, Myenergy, and Ploss, M. (2017). Weiterentwicklung der Gebäuderenovierungsstrategie. Weiterreichende Strategieansätze und Maßnahmen. Governmental Publication, pages 1-38.

Myenergy Luxemgourg (2018). Quelles aides financières pour votre projet de construction ou de rénovation. Myenergy Publication, pages 1-21.

OECD, IEA, NEA, and ITF (2015). Aligning policies for a low-carbon economy. OECD publishing, pages $1-242$.

Olhoff, A. and Christensen, J. M. (2018). Emissions gap report 2018. U.N. Environment Program, pages 1-112.

Pachauri, R. K., Allen, M. R., Barros, V. R., Broome, J., Cramer, W., Christ, R., Church, J. A., Clarke, L., Dahe, Q., Dasgupta, P., et al. (2014). Climate Change 2014: Synthesis Report. Contribution of Working Groups I, II and III to the Fifth Assessment Report of the Intergovernmental Panel on Climate Change. IPCC, pages 1-151.

Palmer, J. A., Suggate, J., Robottom, I., and Hart, P. (1999). Significant life experiences and formative influences on the development of adults' environmental awareness in the UK, Australia and Canada. Environmental Education Research, 5(2):181-200.

Pearce, D. (1991). The role of carbon taxes in adjusting to global warming. The Economic Journal, 101(407):938-948.

Peck, S. C. and Teisberg, T. J. (1992). CETA: A model for carbon emissions trajectory assessment. The Energy Journal, 13:55-77.

Petersdorff, C., Boermans, T., and Harnisch, J. (2006). Mitigation of $\mathrm{CO}_{2}$ emissions from the EU-15 building stock. Beyond the EU directive on the energy performance of buildings. Environmental Science and Pollution Research, 13(5):350-358.

Pizer, W. A. (2002). Combining price and quantity controls to mitigate global climate change. Journal of Public Economics, 85(3):409-434. 
Poncin, S. (2019). Energy policies for eco-friendly households in Luxembourg-a study based on the LuxHEI model. Open Repository and Bibliography, pages 1-50.

Reynolds, T. W., Bostrom, A., Read, D., and Morgan, M. G. (2010). Now what do people know about global climate change? Survey studies of educated laypeople. Risk Analysis: An International Journal, 30(10):1520-1538.

Rivers, N. and Jaccard, M. (2005). Combining top-down and bottom-up approaches to energyeconomy modeling using discrete choice methods. The Energy Journal, 26:83-106.

Rohdin, P. and Thollander, P. (2006). Barriers to and driving forces for energy efficiency in the non-energy intensive manufacturing industry in Sweden. Energy, 31(12):1836-1844.

Sartori, I., Wachenfeldt, B. J., and Hestnes, A. G. (2009). Energy demand in the Norwegian building stock: Scenarios on potential reduction. Energy Policy, 37(5):1614-1627.

Schaefer, C., Weber, C., Voss-Uhlenbrock, H., Schuler, A., Oosterhuis, F., Nieuwlaar, E., Angioletti, R., Kjellsson, E., Leth-Petersen, S., Togeby, M., et al. (2000). Effective policy instruments for energy efficiency in residential space heating: An international empirical analysis (EPISODE). Institut für Energiewirtschaft und Rationelle Energieanwendung, pages 1-123.

Schimschar, S., Blok, K., Boermans, T., and Hermelink, A. (2011). Germany's path towards nearly zero-energy buildings-Enabling the greenhouse gas mitigation potential in the building stock. Energy Policy, 39(6):3346-3360.

Schleich, J. and Gruber, E. (2008). Beyond case studies: Barriers to energy efficiency in commerce and the services sector. Energy Economics, 30(2):449-464.

Schleussner, C.-F., Rogelj, J., Schaeffer, M., Lissner, T., Licker, R., Fischer, E. M., Knutti, R., Levermann, A., Frieler, K., and Hare, W. (2016). Science and policy characteristics of the Paris Agreement temperature goal. Nature Climate Change, 6(9):827.

Schofer, E. and Meyer, J. W. (2005). The worldwide expansion of higher education in the twentieth century. American Sociological Review, 70(6):898-920.

Schulz, M., and Mavroyiannis, A. D. (2012). Directive 2012/27/EU of the European Parliament and of the Council of 25 October 2012 on energy efficiency, amending Directives 2009/125/EC and 2010/30/EU and repealing Directives 2004/8/EC and 2006/32/EC. Official Jorunal of the European Union, pages 1-55.

Simon, H. A. (1955). A behavioral model of rational choice. The Quarterly Journal of Economics, 69(1):99-118.

Sorrell, S., Schleich, J., Scott, S., O'Malley, E., Trace, F., Boede, U., Ostertag, K., and Radgen, P. (2000). Reducing barriers to energy efficiency in public and private organizations. Science and Policy Technology Research (SPRU), pages 1-197.

STATEC (2011). Recensement de la Population.

STATEC (2017). Registre des Bâtiments et des Logements (RBL).

Sunikka-Blank, M. and Galvin, R. (2012). Introducing the prebound effect: the gap between performance and actual energy consumption. Building Research \& Information, 40(3):260-273. 
Thollander, P. and Ottosson, M. (2008). An energy efficient Swedish pulp and paper industryexploring barriers to and driving forces for cost-effective energy efficiency investments. Energy Efficiency, 1(1):21-34.

TIR Consulting Group LLC and Grand Duchy of Luxembourg Working Group (2016). The Third Industrial Revolution Strategy Study for the Grand Duchy of Luxembourg. Governmental Publication, pages 121-149.

Tommerup, H. and Svendsen, S. (2006). Energy savings in Danish residential building stock. Energy and Buildings, 38(6):618-626.

Train, K. (1985). Discount rates in consumers' energy-related decisions: A review of the literature. Energy, 10(12):1243-1253.

Trianni, A. and Cagno, E. (2012). Dealing with barriers to energy efficiency and SMEs: Some empirical evidences. Energy, 37(1):494-504.

Vollebergh, H. (2014). Green tax reform: Energy tax challenges for the Netherlands. $P B L$ Netherlands Environmental Assessment Agency, pages 1-50.

Weber, L. (1997). Some reflections on barriers to the efficient use of energy. Energy Policy, 25(10):833-835.

Weisbach, D. A. (2012). Carbon taxation in the EU: Expanding the EU carbon price. Journal of Environmental Law, 24(2):183-206.

Weiss, J., Dunkelberg, E., and Vogelpohl, T. (2012). Improving policy instruments to better tap into homeowner refurbishment potential: Lessons learned from a case study in Germany. Energy Policy, 44:406-415.

Weiss, M., Junginger, M., Patel, M. K., and Blok, K. (2010). A review of experience curve analyses for energy demand technologies. Technological Forecasting and Social Change, 77(3):411-428.

Weitzman, M. L. (1974). Prices vs. quantities. The Review of Economic Studies, 41(4):477-491. 